IIUL IISER

LUXEMBOURG InSIITUTE OF

SOCIO-ECONOMIC RESEARCH 
LISER Working Papers are intended to make research findings available and stimulate comments and discussion. They have been approved for circulation but are to be considered preliminary. They have not been edited and have not been subject to any peer review.

The views expressed in this paper are those of the author(s) and do not necessarily reflect views of LISER. Errors and omissions are the sole responsibility of the author(s). 


\title{
Labor Market Sorting: \\ The Medium-Term Economic Impact of COVID-19
}

\author{
Michał Burzyński* \\ Luxembourg Institute of Socio-Economic Research (LISER)
}

November 17, 2020

\begin{abstract}
This paper conducts a quantification of economic consequences of COVID-19 crisis for the Luxembourgish economy. We build a general equilibrium model that incorporates firms', goods' and labor markets, as well as interactions across them. The innovativeness of our theory comes from endogenizing workers' sorting across multiple occupations and sectors. Moreover, we derive closed-form solutions for occupation-specific equilibrium wage distributions, and we numerically compute occupation-sector-specific wage distributions. We find that the COVID-19 shock has a strong distributive effect across sectors and occupations: the self-selection of workers deteriorates in sectors experiencing negative fallout, and vice versa. Overall, the total GDP and employment are adversely affected, while the inequality in the whole economy and within many sectors increases.
\end{abstract}

Keywords: COVID-19, Occupational Sorting, Comparative Advantage, Wage Distribution, Inequality, General Equilibrium

JEL classification: C68, J24, J31, J62, L11.

\footnotetext{
*Contact: Michał Burzyński: michal.burzynski@liser.lu. The paper is a part of the 14854549 - MODVid project funded by the FNR Luxembourg COVID-19 Fast Track-2 Call. The paper benefited from a fruitful cooperation between LISER and LuxInnovation. In particular, I would like to thank Maxime Delahaut and Sara Bouchon for their priceless help on providing firm-level data used for one of the model simulations.
} 


\section{Introduction}

The economic crisis caused by COVID-19 generates unprecedented pressure on all economic agents: workers, entrepreneurs, and political authorities. The initial spread of the disease and the consequent fast reactions of numerous governments to lock national economies have put many countries in recessions of magnitudes unseen for almost a century. The short-term implications of the COVID-19 economic crisis are already well documented in data. Many developed economies experienced huge falls in national gross domestic products, followed by temporary reductions in employment, mitigated as economies reopened. Firms started to face liquidity problems, despite early fiscal interventions of governments. The questions that remain unanswered are those that look beyond the short-term perspective: Which sectors and occupations will experience the highest outflows of workers? How will unemployment be affected? What adjustments in prices and wages can we expect after the markets settle? What if vulnerable firms will be forced to quit the market? What would happen, if people refrain from consuming specific goods and services and the pandemic lasts several more months? The quantification of the medium-term economic consequences of these phenomena is the goal of this paper.

Our approach includes a multi-sector general equilibrium model with endogenous consumption decisions, entry and exit of firms, as well as a detailed national labor market composed of sector-occupation-specific cells. The modeling choice is motivated by its ability to jointly investigate the interactions and spillovers across goods', firms', and labor markets in a unified framework. Our quantitative results focus on three crucial mechanisms induced by the COVID-19 recession. First, we study changes in consumption patterns. Various studies (e.g. Andersen et al., 2020; Baker et al., 2020) demonstrate a sharp shift in peoples' expenditures, induced primarily by mobility constraints and economic lockdowns. Sectoral heterogeneity in these changes is immense, therefore sector-specific implications are bound to be strongly differentiated. Second, we investigate the consequences of potential waves of firm bankruptcies - an exogenously determined limitation on the labor demand brought about, most probably, by higher running costs and liquidity problems (Guerini et al., 2020; Demmou et al., 2020; Carletti et al., 2020; De Vito and Gomez, 2020). Losses in terms of numbers of operating firms are believed to generate the most threatening spillover across all interconnected markets, inflating forced unemployment and constraining the supply of goods and services. Certainly, these phenomena will strongly be related to the sectoral activity, as the distribution of key firm characteristics (productivity, size, market power, profitability, risk level) is strongly sector-specific. Finally, our model takes an innovative approach towards quantifying labor market impacts 
of the COVID-19 recession, by allowing people to switch occupations and be reassigned across various production sectors. We internalize that labor mobility decisions are dictated not only by crude changes in expected wages and the supply of production factors, but most importantly by outside options that heterogeneous workers face on a specific labor market. These options, by determining mobility constraints in an indirect way, emerge from the matching between peoples' skills and tasks that are demanded by active firms. A mismatch between the two pushes some workers into unemployment, while other employees reap the gains from skill transferability and decide to join growing sectors. Our model generates multidimensional selection and sorting patterns across occupations and sectors, which fundamentally enriches the macroeconomic message that we put forward. We believe that encompassing these three modules of the economy yields a comprehensive, yet a detailed theoretical setup that is highly relevant for studying the impact of COVID-19 on the national economy and consequential policy interventions.

We propose a handful of simulations that mimic the economic fallout of the COVID-19 crisis, accounting for sectoral heterogeneity in general equilibrium adjustments. Two of them implement exogenous demand shocks in sectors that are most vulnerable to COVID19 recession, namely low-skill services and sales. In these cases, we analyze the model mechanics and verify the economy's resilience to sector-specific shocks. The last scenario is defined using macroeconomic data on the Luxembourgish economy. We exogenously impose a change in individual preference parameters that corresponds to changes in the structure of demand in Luxembourg in the second quarter of 2020, published by Eurostat. Moreover, we translate these observed shifts in the demand into shocks on the firms' side, calibrated using Chambre de Commerce Luxembourg and Editus data. As their turnover drops, some firms might experience liquidity problems. Nonetheless, all firms face higher costs of running the business (due to social distancing or increased hygiene measures). In this scenario, the main original message is that sectors that experience most severe reductions in demand - sales and low-skill services - also lose the most efficient workers. Crucially, in these "losing" sectors, the selection of employees deteriorates, as the most skilled employees choose to switch to more prosperous sectors within the same job type. However, we also report a visible labor shift across occupations, as jobs intensively exploited in losing sectors - service and elementary occupations - experience outflows of workers in the whole economy. As these are predominantly blue-collar workers, who dispose of limited outside options, their outflows are mainly directed to unemployment. On the contrary, sectors that win from the COVID-19 crisis, e.g. transportation or professional services, grow in employment, improve worker quality, augment the number of incumbent firms and their average productivity. Wage distributions in these sectors im- 
prove, but mainly those located in right tails observe gains. In aggregated terms, workers in blue-collar-intensive sectors lose (either due to lower wages or due to unemployment), while white-collar intensive sectors gain (mainly by executing their outside options in moving across sectors and occupations). This generates tensions on the labor market, which cause uneven distributions of losses induced by the COVID-19 crisis: inequality increases visibly even when worker mobility is allowed and workers shuffle across the labor market. In total, the GDP in Luxembourg falls permanently by $4.8 \%$ in the new general equilibrium, whereas the unemployment rate rises by $33 \%$.

In technical terms, our setup joins and extends the literature on self-selection, assignment, and general equilibrium models. The structure of labor market builds strongly on the work of Roy (1951); Heckman (1979); Heckman and Sedlacek (1985); Heckman and Honore (1990). We assume that workers are endowed with multidimensional skill vectors that are distributed according to an $N$-dimensional log-Normal, each skill can be used in one job type (occupation) only. As people sort across $N$ occupations executing the comparative advantage in skills they possess, and for given skill market prices, the equilibrium allocation of workers sets up a multidimensional Roy-type structure. We solve for the equilibrium worker allocations and wage distributions in each of $N=5$ market occupations, applying the findings of Azzalini (1985); Azzalini and Valle (1996); Azzalini (2005); Arellano-Valle and Azzalini (2006). Then, within each occupation, we allow workers to be assigned across $S=8$ sectors. Sectors are heterogeneous in terms of production technology and the intake of occupation-specific tasks. Workers and firms agree on a common ranking of sectors, and draw idiosyncratic shocks which determine employees' placement across sectors. In this way, our model generates forty continuous sector-occupation-specific distributions of wages as an endogenously determined output. The demand for labor is governed by Cobb-Douglas production technology, including a capital and labor composite, the latter being a complementary function of occupationspecific tasks. Continuously heterogeneous firms operate in a monopolistically competitive market, as in Melitz (2003). This implies free entry and zeroing of cutoff profits, which determine the equilibrium mass and productivity of incumbent firms.

Our model necessarily refers to the flourishing literature on labor market self-selection models as Burstein et al. (2020). Clearly, we cannot achieve the algebraical tractability of the Fréchet-based models (even though we derive the analytical solution for the equilibrium wage distributions). However, the proposed model does not suffer from the common critique of the self-selection models based on extreme value distributions with independent draws of idiosyncratic shocks. In particular, the conditional and unconditional distributions of wages are not identical in the equilibrium, which is the case in 
labor markets in which worker productivity is drawn from any Extreme Value Distribution. Thus, with our approach, we are able to study more complex dynamics of labor mobility across occupations, with higher moments of the equilibrium distributions being affected by self-selection (meaning: who decides to moves away from their current job) and sorting (meaning: to which job movers decide to switch).

The rest of the paper is organized as follows. Section 2 provides a detailed description of the theory, including producers' and consumers' decisions, labor market sorting, aggregation and equilibrium. Then, in Section 3, we discuss the numerical analysis: data inputs, identification strategy, and the calibration process. Section 4 discusses simulation results, while Section 5 concludes.

\section{Theoretical model}

In this section, we set up the basic structure of our theoretical model. We commence with a high-level description of each module, and a preview of the general equilibrium (GE). The theoretical analysis starts with defining the economic environment and determining the sector-specific production functions. We continue with specifying peoples' consumption decisions and we define the goods' market equilibrium. Then, we move to describing the firms' decisions and we define the equilibrium market size. Finally, we provide a detailed discussion of the labor market, followed by the definitions of labor market equilibrium and the general equilibrium.

\subsection{Economic Environment}

Market structure Assume that the national economy is a single geographical unit partitioned into $s \in S$ sectors. Each sector is composed of infinitely many firms that operate on a monopolistically competitive market, as in Melitz (2003). This implies that: (i) in every sector $s$, firms produce differentiated $s$-specific goods, (ii) firms are ex-ante identical, and decide to enter the market by comparing expected profits with sectorspecific entry costs, (iii) after the entry, firms randomly draw their specific productivity level and decide whether to stay or leave the market, (iv) the free entry condition equalizes expected profits with entry costs, (v) the zero profit condition equalizes the profits of least productive firms to zero.

Production The production process in every sector $s$ requires an input of capital and tasks performed by heterogeneous workers, aggregated into a Cobb-Douglas production 
function. We assume that the capital stock adjusts whereas the equilibrium interest rate is constant. Similarly, labor adjusts and allocates across tasks to achieve the equilibrium wage rates. In particular, tasks are carried out by workers assigned to a specific occupation $n \in N$. Depending on its specificity, each sector has its own Cobb-Douglas structure of occupational inputs, determining the required combination of efficient labor units that have to be filled across sectoral occupations. The value added produced in sector $s$ equals the sum of units produced multiplied by the sector-specific prices. The gross domestic product (GDP) of the economy aggregates nominal values of outputs for all $s \in S$.

Labor Market Workers are characterized by multidimensional skills that determine their productivity across occupations. After having formed expectations about average wage rates payed in various occupations, individuals sort across occupations, following the principle of comparative advantage. Then, according to idiosyncratic shocks, workers select the sector to join, conditional on their previous occupational choice. Overall, the model aims at studying workers' endogenous assignment across multiple occupations and sectors (the Cartesian product of which will be called a set of labor market cells).

Equilibrium The general equilibrium (GE) of the model comprises of four elements: (i) workers sort across occupations and sectors by maximizing their expected utility (equivalent to maximizing wage incomes), (ii) firms minimize costs and rationally enter/exit the market in each sector, which exercises the free entry and the zero profit conditions, (iii) people spend all their income on consumption, given their preferences for sectorspecific goods, which determines equilibrium prices, (iv) labor markets clear, as wages equalize marginal productivities of sector-occupational tasks inputs.

\subsection{Producers' Perspective}

Let $Y=\sum_{s \in S} Y_{s}$ be the total nominal GDP created in all sectors of the economy. From the production perspective, sectoral GDPs equal marginal costs multiplied by quantities of goods produced: $Y_{s}=c_{s} \bar{Q}_{s}$. Note that the number of produced units depends on the factors employed (capital and efficient task supply), and takes the Cobb-Douglas form: $\bar{Q}_{s}=\beta_{s} K_{s}^{\theta_{s}} L_{s}^{1-\theta_{s}}$, with aggregated productivity and factor income shares determined at the sectoral level. Hence, in every $s \in S$, firms face a marginal cost of production: $c_{s}=\beta_{s}^{-1}\left(i_{s} / \theta_{s}\right)^{\theta_{s}}\left(w_{s} /\left(1-\theta_{s}\right)\right)^{1-\theta_{s}}$, where $i_{s}$ and $w_{s}$ are market prices for capital and completing tasks (taken by firms as given). The market clearing conditions require that factor remunerations sum up to sectoral GDPs: $Y_{s}=w_{s} L_{s}+i_{s} K_{s}$. 
In each sector $s$, the demand for efficient task input, $L_{s}$, can be decomposed into tasks fulfilled by people working in specific occupations. Every occupation is responsible for completing a given subset of tasks, and only those workers, who are assigned to this occupation are allowed to fulfill them. The structure of tasks within sectors is given, and imposes a predefined structure of basic inputs for production. Assume that there exist $N$ distinct occupations indexed by $n$. Then, the aggregated sectoral task input takes a form of a Cobb-Douglas function:

$$
L_{s}=\prod_{n \in N} L_{s, n}^{\gamma_{s, n}}
$$

where $\gamma_{s, n}$ is the share of labor output value that can be attributed to occupation $n$ in sector $s$. By defining a multiplicative production function of sector-specific goods, we impose a strong complementarity between outputs of various occupations. In particular, as long as $\forall s, n \gamma_{s, n}>0$, it is necessary to fulfill all relevant tasks to produce a unit of value added, unconditionally on who performs these tasks. The market price of completing each bundle of tasks $n$ in sector $s$ equals:

$$
w_{s, n}=\beta_{s} \gamma_{s, n}\left(1-\theta_{s}\right) Y_{s} / L_{s, n},
$$

where $L_{s, n}$ is the sector-occupation-specific efficient labor composite, that is: the sum of effective skills of workers, who decided to join a labor market cell $(s, n)$.

\subsection{Consumers' Perspective}

All individuals spend their earned income on goods produced within sectors $s \in S$. However, in their optimal choices, people internalize double heterogeneity of consumption goods. Apart from sectoral specificity, goods are differentiated within sectors, as we assume that firms operate under monopolistic competition. Thus, it seems instructive to divide the consumption problem into outer and inner decisions.

Outer Problem First, people determine the sectoral spendings by maximizing the utility from aggregated consumption. Everyone solves:

$$
\max _{Q_{s}} Q=\left(\sum_{s} \alpha_{s} Q_{s}^{\frac{\varepsilon-1}{\varepsilon}}\right)^{\frac{\varepsilon}{\varepsilon-1}} \quad \text { s.t. } \quad \sum_{s} P_{s} Q_{s}=Y,
$$

in which we assume that each individual has identical, constant elasticity of substitution (CES) preferences with common sectoral shifters $\alpha_{s}$ and the elasticity of substitution 
between sectoral goods equals $\varepsilon$. The solution of this program yields sector-specific demands for real production, and determines the overall price index $P$ as a function of sector-specific characteristics:

$$
Q_{s}=Q\left(\alpha_{s} P / P_{s}\right)^{\varepsilon}, \quad P=\left(\sum_{s} \alpha_{s}^{\varepsilon} P_{s}^{1-\varepsilon}\right)^{\frac{1}{1-\varepsilon}}
$$

Inner Problem After solving the outer problem, consumers determine the structure of consumption within each sector. They realize that the mass of firms in sector $s \in S$ offers an uncountable array of differentiated goods, which can be ordered by $\psi_{s} \in \Psi_{s}$, so that the problem reduces to:

$$
\max _{q_{s}\left(\psi_{s}\right)} Q_{s}=\left(\int_{\psi_{s} \in \Psi_{s}} q_{s}\left(\psi_{s}\right)^{\frac{\sigma-1}{\sigma}} d \psi_{s}\right)^{\frac{\sigma}{\sigma-1}} \quad \text { s.t. } \quad \int_{\psi_{s}} p_{s}\left(\psi_{s}\right) q_{s}\left(\psi_{s}\right)=P_{s} Q_{s} .
$$

Note that varieties of sectoral goods are also assumed to be imperfect substitutes, as people reveal CES preferences with a constant elasticity of substitution equal to $\sigma$. In analogy to the solution of the outer problem, we can write that optimal demand for each variety $\psi_{s} \in \Psi_{s}$ equals:

$$
q_{s}\left(\psi_{s}\right)=Q_{s}\left(P_{s} / p_{s}\left(\psi_{s}\right)\right)^{\sigma}, \quad P_{s}=\left(\int_{\psi_{s}} p_{s}\left(\psi_{s}\right)^{1-\sigma}\right)^{\frac{1}{1-\sigma}}
$$

The latter pair of expressions gives us a standard definition of equilibrium on goods' market:

Definition 1. Take any sector $s \in S$. For a given vector of individual preferences $\alpha_{s}$, income $Y_{s}$, and a collection of prices set by firms $p_{s}\left(\psi_{s}\right)$, the goods' market is in equilibrium if and only if the demand for any good $\psi_{s}$ and its supply coincide: (D1) $q_{s}\left(\psi_{s}\right)=Q_{s}\left(P_{s} / p_{s}\left(\psi_{s}\right)\right)^{\sigma}$.

\section{$2.4 \quad$ Firms}

As in Melitz (2003), active firms have to pay a common, sector-specific fixed cost of production $f_{s}$. Firms are, however, differentiated with respect to variable costs of production (hence: productivity), $\varphi_{s}$. Being local monopolists, and thus knowing the demand curve for its products, firms set the optimal price equal to a constant markup over the marginal cost of production. Recalling that the sector-specific marginal cost, $c_{s}$, originates from a 
two-factor Cobb-Douglas production function, the optimal pricing of $\varphi_{s}$ firm follows:

$$
p_{s}\left(\varphi_{s}\right)=\frac{\sigma}{\sigma-1} \cdot \frac{c_{s}}{\varphi_{s}}
$$

as a result of a standard profit maximization program. Consequently, as in Melitz (2003), we can write the descriptives of each $s$-specific firm (total profit, revenues, and production sold, respectively): ${ }^{1}$

$$
\begin{aligned}
& \pi_{s}\left(\varphi_{s}\right)=R_{s}\left(\varphi_{s}\right) / \sigma-f_{s} \\
& R_{s}\left(\varphi_{s}\right)=Y_{s}\left(\frac{\sigma-1}{\sigma} \cdot \frac{\varphi_{s} P_{s}}{c_{s}}\right)^{\sigma-1} \\
& q_{s}\left(\varphi_{s}\right)=Q_{s}\left(\frac{\sigma-1}{\sigma} \cdot \frac{\varphi_{s} P_{s}}{c_{s}}\right)^{\sigma} .
\end{aligned}
$$

Entry and Exit Assume that there is an infinite supply of potential entrepreneurs, who have to pay a sector-specific entry cost, $\bar{f}_{s}$, to be able to draw their productivity level $\varphi_{s}$ from a given distribution $G_{s}\left(\varphi_{s}\right)$ (with a probability density function (PDF) of $\left.g_{s}\left(\varphi_{s}\right)\right)$. Let the distribution of $s$-specific firm productivities conditional on successful entry be $\mu_{s}\left(\varphi_{s}\right)$, while the mass of firms that end up producing in sector $s$ equals $M_{s}$. The zero profit condition imposes that, conditional on entering, no firm prefers to earn negative profits, which generates a natural cutoff $\varphi_{s}^{*}$ on productivity level: $\pi_{s}\left(\varphi_{s}^{*}\right)=0 \leftrightarrow$ $f_{s}=R_{s}\left(\varphi_{s}^{*}\right) / \sigma$. Consequently, we can derive the relationship between unconditional and conditional distribution of firms' productivity as:

$$
\mu_{s}\left(\varphi_{s}\right)=\frac{g_{s}\left(\varphi_{s}\right)}{1-G_{s}\left(\varphi_{s}^{*}\right)}, \text { for } \varphi_{s} \geq \varphi_{s}^{*}, \quad 0 \text { otherwise }
$$

noting that $1-G_{s}\left(\varphi_{s}^{*}\right)$ is the fraction of firms that decide to remain on the market in sector $s$. Finally, the free entry condition yields that the average expected profit over the life of a successful entering firm has to equal the entry cost:

$$
\left(1-G_{s}\left(\varphi_{s}^{*}\right)\right) v_{s}\left(\bar{\varphi}_{s}\right)=\bar{f}_{s}
$$

where: $v_{s}\left(\bar{\varphi}_{s}\right)=\sum_{t}\left(1-d_{s}\right)^{t}\left(1-\rho_{s}\right)^{t} \max \left(\pi_{s}\left(\bar{\varphi}_{s}\right), 0\right)$ is the expected value of discounted profits of the firm in sector $s$ that possesses the average level of productivity $\bar{\varphi}_{s}$. Expectation includes that firms are subject to an exogenous probability of bankrupting,

\footnotetext{
${ }^{1}$ Note that for any two $\varphi_{1}, \varphi_{2}$, it holds that: $R_{s}\left(\varphi_{1}\right) / R_{s}\left(\varphi_{2}\right)=\left(\varphi_{1} / \varphi_{2}\right)^{\sigma-1}$ and $q_{s}\left(\varphi_{1}\right) / q_{s}\left(\varphi_{2}\right)=$ $\left(\varphi_{1} / \varphi_{2}\right)^{\sigma}$.
} 
$\rho_{s}$. Discounting is done using the rate $d_{s}$. Overall, we simplify notation by setting: $\delta_{s}=d_{s}+\rho_{s}-d_{s} \rho_{s}$, so that: $\forall \varphi_{s} v_{s}\left(\varphi_{s}\right)=\max \left(\pi_{s}\left(\varphi_{s}\right) / \delta_{s}, 0\right)$.

Distribution of Productivity The final detail of the firms' module that remains to be determined before providing an analytical definition of the equilibrium, is the functional form for the distribution of productivities. Assume a Pareto distribution with a sector-specific shape parameter $\lambda_{s}$, and a common scale parameter $\varphi_{0}$ that determines the minimal level of productivity in each sector:

$$
G_{s}\left(\varphi_{s}\right)=1-\left(\varphi_{0} / \varphi_{s}\right)^{\lambda_{s}} \quad g_{s}\left(\varphi_{s}\right)=\lambda_{s} \varphi_{0}^{\lambda_{s}} \varphi_{s}^{-\lambda_{s}-1}
$$

hence, the conditional density of active firms writes $\mu_{s}\left(\varphi_{s}\right)=\lambda_{s}\left(\varphi_{s}^{*}\right)^{\lambda_{s}} \varphi_{s}^{-\lambda_{s}-1}$. We can now compute the average productivity level in sector $s$ :

$$
\bar{\varphi}_{s}=\left(\int_{\varphi_{s}^{*}}^{\infty} \varphi_{s}^{\sigma-1} \mu_{s}\left(\varphi_{s}\right) d \varphi_{s}\right)^{\frac{1}{\sigma-1}} \rightarrow\left(\frac{\bar{\varphi}_{s}}{\varphi_{s}^{*}}\right)^{\sigma-1}=\frac{\lambda_{s}}{\lambda_{s}-(\sigma-1)}
$$

showing that the ratio of average and cutoff productivities is constant, which is an inherent property of the Pareto distribution. Importantly, the derived relationship makes sense only when $\lambda_{s}>\sigma-1$.

Equilibrium Conditions We can rewrite the sectoral characteristics averaging across all active firms in $s$. One can show, as elaborated in Melitz (2003), that sectoral price indexes reduce to: $P_{s}=M_{s}^{\frac{1}{1-\sigma}} p_{s}\left(\bar{\varphi}_{s}\right)$, while the total GDP can be decomposed into: final goods' production, fixed costs investments and total profits (equal to entry costs investments):

$$
Y_{s}=M_{s} c_{s} z\left(\bar{\varphi}_{s}\right)+M_{s} f_{s}+\Pi_{s}
$$

where: $z\left(\bar{\varphi}_{s}\right)$ is the demand for efficient composite (recall it is a Cobb-Douglas of efficient labor and capital) by the firm with average productivity for the purpose of producing final goods, $z\left(\bar{\varphi}_{s}\right)=q_{s}\left(\bar{\varphi}_{s}\right) / \bar{\varphi}_{s}$, while $\Pi_{s}$ is the sum of profits earned by firms in sector $s$, such that:

$$
\Pi_{s}=M_{s} \pi\left(\bar{\varphi}_{s}\right)=Y_{s} / \sigma-M_{s} f_{s}
$$

since $\pi\left(\bar{\varphi}_{s}\right)=R_{s}\left(\bar{\varphi}_{s}\right) / \sigma-f_{s}$, and the total turnover of firms equals GDP: $M_{s} R_{s}\left(\bar{\varphi}_{s}\right)=Y_{s}$. Thus, we have that: $M_{s} c_{s} z\left(\bar{\varphi}_{s}\right)=\frac{\sigma-1}{\sigma} Y_{s}$, which multiplied by $\sigma /(\sigma-1)$ gives us a measure of average production in terms of units of goods in sector $s$, as well as the demand for efficient component, including production and investment purposes that constitute 
$(\sigma-1) / \sigma$ and $1 / \sigma$ shares of GDP, respectively:

$$
\frac{\sigma}{\sigma-1} q_{s}\left(\bar{\varphi}_{s}\right)=\frac{\bar{\varphi}_{s} Y_{s}}{c_{s} M_{s}}, \quad \bar{Q}_{s}=\frac{\sigma}{\sigma-1} M_{s} z\left(\bar{\varphi}_{s}\right)=\frac{Y_{s}}{c_{s}}=\beta_{s} K_{s}^{\theta_{s}} L_{s}^{1-\theta_{s}} .
$$

Now, one can join the producers' and consumers' accounting of GDP, which gives:

$$
P_{s} Q_{s}=Y_{s}=c_{s} \bar{Q}_{s} \rightarrow Q_{s}=\frac{\sigma-1}{\sigma} \bar{Q}_{s} M_{s}^{\frac{1}{\sigma-1}} \bar{\varphi}_{s}=q_{s}\left(\bar{\varphi}_{s}\right) M_{s}^{\frac{\sigma}{\sigma-1}}
$$

or equivalently:

$$
P_{s}=M_{s}^{\frac{1}{1-\sigma}} p_{s}\left(\bar{\varphi}_{s}\right)=M_{s}^{\frac{1}{1-\sigma}} \frac{\sigma}{\sigma-1} \frac{c_{s}}{\bar{\varphi}_{s}} .
$$

Consequently, GDP accounting postulates equalization of income, value of demand, value of production, and firms' revenues. Taking the equivalence of the first and the last element in the chain, that is: $Y_{s}=M_{s} R_{s}\left(\bar{\varphi}_{s}\right)$, coupled with $R_{s}\left(\varphi_{1}\right) / R_{s}\left(\varphi_{2}\right)=\left(\varphi_{1} / \varphi_{2}\right)^{\sigma-1}$ for any two $\varphi_{1}, \varphi_{2}$ we can rewrite the zero profit condition as:

$$
R_{s}\left(\bar{\varphi}_{s}^{*}\right)=\sigma f_{s} \rightarrow M_{s}=\frac{Y_{s}}{\sigma f_{s}}\left(1-\frac{\sigma-1}{\lambda_{s}}\right)
$$

which is equivalent to a constant profit share in sector $s$ :

$$
\frac{\Pi_{s}}{Y_{s}}=\frac{\sigma-1}{\lambda_{s} \sigma} .
$$

Finally, we can rewrite the free entry condition using explicit functions for average profits, $\pi_{s}\left(\bar{\varphi}_{s}\right)$, and the probability of staying on the market, $1-G_{s}\left(\varphi_{s}^{*}\right)=\left(\varphi_{0} / \varphi_{s}^{*}\right)^{\lambda_{s}}$ :

$$
\left(\frac{\varphi_{0}}{\varphi_{s}^{*}}\right)^{\lambda_{s}}=\frac{\delta_{s} \bar{f}_{s}}{f_{s}}\left(\frac{\lambda_{s}}{\sigma-1}-1\right) .
$$

To complete the exposition of equilibrium equations, we mention the dynamic consistency condition, which equalizes the fraction of successful survivors among the new entries into each industry, $M_{s}^{e}$, with the number of exits: $\left(\varphi_{0} / \varphi_{s}^{*}\right)^{\lambda_{s}} M_{s}^{e}=\rho_{s} M_{s}$.

Definition 2. Take any sector $s \in S$. For given factor prices and incomes, allocations of factors $K_{s}, L_{s}$, substitutability of consumption goods $\sigma$, fixed and entry costs, and the distribution of productivities $G_{s}\left(\varphi_{s} ; \varphi_{0}, \lambda_{s}\right)$, the equilibrium firms' market size is characterized by:

(D2) the mass of entering firms deriving from the Zero Profit Condition, Equation (18),

(D3) the cutoff productivity deriving from the Free Entry Condition, Equation (20). 


\subsection{Labor Market}

Our approach towards modeling the labor market roots in the setup of Roy (1951), but extends it in several aspects. First, occupation-specific skills are log-normally distributed in the population of workers. However, we choose to write up the model in $N>2$ dimensions, which introduces considerable complexity into deriving the analytical solution of the model. Second, as in Roy (1951), workers sort across occupations by maximizing their expected utility and following the principle of comparative advantage. Third, given their choices regarding occupational sorting, workers select their sector of activity, which is guided by idiosyncratic shocks. Fourth and finally, our approach includes crucial interactions between labor supply and labor demand including consumption externalities and distributions of firms' productivity, which strongly extends the partial equilibrium perspective analyzed so far into a GE environment.

Consolidating previous explanations, the labor market analyzed in our model is twodimensional: sectors cross with occupations, which essentially impacts workers' assignment to tasks. From the firms' perspective, the sectoral dimension is the dominant one: firms operate in sectors, and in each $s \in S$ they need to fill a given structure of tasks (performed by workers employed in specific occupations). From the workers' perspective, occupational dimension leads the allocation decisions, as people first choose the type of job they want to pursue (optimized conditional on workers' skills and abilities), as different occupations require different skills. Knowing their general field of expertise (occupation), they decide which sector to join, following the realizations of idiosyncratic shocks. Note, however, that the same occupations in different sectors require the same skill sets, so that the only criterion of sorting across sectors within a given occupation are unobservable shocks.

Occupational Sorting Assume that every individual is endowed with a given bundle of skills $\mathbf{y} \in \mathbb{R}_{+}^{N}$. There are $N$ distinct skills that enable workers to fulfill tasks in $N$ different occupations. Each of $N$ occupations is characterized by specific tasks that can be completed by workers using respective $N$-specific skills. The log return from supplying each of $N$ skills writes:

$$
w_{n}\left(y_{n}\right)=\xi_{n}+\omega_{n} y_{n}, n \in 1, \ldots, N \text {. }
$$

In the equation above, $\xi_{n}$ reflects the expected marginal output from a unit of $n$-specific skill, a term identical for all workers. In contrast, $y_{n}$ is individual-specific, and represents personal stock of skill $n$. People are heterogeneous with respect to their skill endowments, and we assume that $\left(y_{1}, \ldots, y_{N}\right)$ are distributed in the population of all workers according 
to $\mathcal{N}(0, \Omega)$, where $\Omega$ is a correlation matrix with off-diagonal entries labeled by $\omega_{n m}$. The terms $\omega_{n}$ stand for standard deviations of respective marginal skill distributions. ${ }^{2}$

In absence of workers' sorting, observed wage distributions resulting from Equation (21) would be log-normal. Individuals, however, select one and only one occupation in which they are active, and these choices are determined by market returns to skills. Everyone strives at maximizing their gain from supplying occupation-specific skills, thus sorting is determined by workers' comparative advantage in completing one of $N$ tasks. As workers who decide to be active in occupation $n$ are a non-random subsample of the workforce, the self-selection bias alters all the moments of the observed wage distributions. Formally, people solve their wage maximization program: $\max _{n \in N} w_{n}\left(y_{n}\right)$. The latter implies that the post-sorting wage distributions across occupations equal to:

$$
W_{n}(\mathbf{y})=\left(w_{n}(\mathbf{y}) \mid \forall_{m \neq n} w_{n}(\mathbf{y})>w_{m}(\mathbf{y})+\kappa_{n}\right),
$$

where $\kappa_{n}$ represents costs of moving away from occupation $n$.

To close the individual-level analysis of occupational sorting, consider the log of expected return to skills, denoted by $\xi_{n}$. As discussed in previous subsections, returns to all factors are equalized to their marginal productivities. Hence, the market prices of completing tasks $n$ in sector $s$ equals to $w_{s, n}$ and is determined by Equation (2). Let $\eta_{s, n}=L_{s, n} / \sum_{s, n} L_{s, n}$ represent the share of workers in labor market cell $(s, n)$. We now simply write the logarithm of expected returns to skills for every occupation $n \in N$ as:

$$
\xi_{n}=\sum_{s \in S} \eta_{s, n} \ln \left(w_{s, n}\right)
$$

This expression joins the supply and demand sides of the labor market, as the left hand side represents the main driving force for workers' sorting, while the right hand side aggregates prices for tasks that depend on consumption demand, technology of production, and the population of active firms.

Aggregation In this part, our aim is to present the analytical solution for the occupationspecific distributions of wages after workers' sorting. To start with, consider some addi-

\footnotetext{
${ }^{2}$ Notice that this specification is equivalent to defining $D$ universal skills $x_{d} \in \mathbb{R}_{+}^{D}$ that are used in $n$ occupations in heterogeneous combination. Formally: $y_{n}=\sum_{d \in D} \zeta_{n, d} x_{d}$, and $x_{d} \sim \mathcal{N}\left(0, \Omega_{d}\right)$ and $\left[\zeta_{n, d}\right]_{N, D}$ is a full rank matrix.
} 
tional notation for any pair of occupations $n, m \in N$ :

$$
\begin{aligned}
y_{n-m} & =\left(\omega_{n} y_{n}-\omega_{m} y_{m}\right) / \omega_{n-m}, \\
\tau_{n-m} & =\left(\xi_{n}-\xi_{m}-\kappa_{n}\right) / \omega_{n-m}, \\
\omega_{n-m} & =\left(\omega_{n}^{2}+\omega_{m}^{2}-2 \omega_{n} \omega_{m} \omega_{n m}\right)^{1 / 2} .
\end{aligned}
$$

The first equation in the set above normalizes the differences in returns to skills between any two occupations. $\tau_{n-m}$ is a result of an analogous manipulation of the common (e.g. market-driven) part of the skill returns. The third equation computes the standard deviation of a random variable $\omega_{n} y_{n}-\omega_{m} y_{m}$. Using this new notation we can rewrite equation (22) in more general terms as:

$$
W_{n}\left(y_{n}\right)=\left(\xi_{n}+\omega_{n} y_{n} \mid \forall_{m \neq n} y_{n-m}+\tau_{n-m}>0\right) .
$$

The latter distribution resembles the one obtained by Heckman and Sedlacek (1985). Formally, it is a uni-dimensional Gaussian distribution conditioned on a set of $N-1$ other (but necessarily dependent) Gaussian distribution. In fact, equation (25) defines a Unified Skew-Normal distribution (labeled by acronym SUN), developed and analyzed by Azzalini and Valle (1996); Azzalini (2005); Arellano-Valle and Azzalini (2006). The SUN distribution can be characterized by five parameters:

$$
W_{n} \sim \mathrm{SUN}_{1, N-1}\left(\xi_{n}, \omega_{n}^{2},\left[\tau_{n}\right]_{N-1},\left[\delta_{n}\right]_{N-1},\left[\Gamma_{n}\right]_{N-1 \times N-1}\right)=\operatorname{SUN}_{1, N-1}\left(\Xi_{n}\right)
$$

The first two elements of the set $\Xi_{n}$ are scalars and equal to the log expected return to skill in occupation $n$, and the variance of skill distribution in the population, as explained in Equation (21). The third element, $\tau_{n} \in \mathbb{R}^{N-1}$, gathers all values of $\tau_{n-m}$ for $m \in\{1, \ldots, N\} \backslash\{n\}$, thus determines the differences in marginal productivities among occupations. The fourth element summarizes correlations between $w_{n}$ and $y_{n-m}$ for all $m \neq n$, so that $\delta_{n} \in[-1 ; 1]^{N-1}$ gathers all values of $\delta_{n-m}$, where:

$$
\delta_{n-m}=\operatorname{cor}\left(w_{n} ; y_{n-m}\right)=\operatorname{cor}\left(\omega_{n} y_{n} ; \omega_{n} y_{n}-\omega_{m} y_{m}\right)=\frac{\omega_{n}^{2}-\omega_{n} \omega_{n m}}{\omega_{n-m}}
$$

Finally, the fifth element of $\Xi_{n}$ gathers information about the correlations across $N-1$ random variables that represent standardized differences in personal skill endowments:

$$
\Gamma_{n m p}=\operatorname{cor}\left(y_{n-m} ; y_{n-p}\right)=\frac{\omega_{n}^{2}-\omega_{n} \omega_{p} \omega_{n p}-\omega_{n} \omega_{m} \omega_{n m}+\omega_{m} \omega_{p} \omega_{m p}}{\omega_{n-m} \omega_{n-p}}
$$


Equation (26) offers a complete specification of the wage distribution observed in each occupation $n$ after sorting. Note that $W_{n}$ depends on: (I) the supply of skills available in the labor market, that is the actual distribution of $\mathbf{y}$; (II) the demand for occupational skills coming from firms that need to complete tasks, represented by the returns to skills $\xi_{n}$, endogenous to demand for goods, technology, and subject to changes in the equilibrium mass of firms; (III) all the outside options that workers have on national labor markets, represented by $\tau_{n}$, which are subject to policy interventions (e.g. through subsidizing re-skilling and reducing occupational mobility wedges); (IV) the actual skill requirements in alternative occupations and the linear relationships across them, represented by $\delta_{n-m}$ and $\Gamma_{n m p}$, which might change over time as skill requirements of tasks evolve. Properties (I)-(IV) will be recalled in the following analysis of the model.

Sectoral Sorting Knowing the occupational choice of workers, and realizing that $N$ occupation-specific wage distributions take the form of a SUN, we can advance with decomposing these distributions into sector-occupation-specific ones. Recall that we assume that within an occupation tasks are identical across sectors. Consequently, after having chosen the optimal occupation, people sort across sectors according to unobservable shocks. The aim of this part is to determine the process of drawing sectoral shocks, and translate them into sorting patterns and wage distributions.

First, let us assume that all workers and firms agree on a common ranking of sectors within each occupation. ${ }^{3}$ This ranking is linear, complete and unique, so that for each occupation $n \in N$, there exists a permutation $r_{n}$ of sector names $s \in S$ that represents common preferences regarding sectors: $r_{n}: S \rightarrow S: \forall_{s_{1}, s_{2} \in S} r_{n}\left(s_{1}\right)>r_{n}\left(s_{2}\right) \leftrightarrow s_{1} \succ s_{2}$. These common preferences might emerge from multiple sources: either by comparing sectoral quality of work, job safety, size of firms, or some other sector-specific characteristics that are not explicitly taken into account in our model. We determine the ranking as a function of expected sectoral wages: sectors with higher average wage rates are unanimously more preferred by all workers in a given occupation $n .{ }^{4}$ Whatever is the true origin of this ranking, we require that people comply to it and are perfectly informed about it. With this ranking in hand, individuals choosing occupation $n$ are partitioned across all sectors, and the shares of workers ordered by increasing ranking write: $\eta_{n} \equiv\left(\eta_{r_{n}^{-1}(1), n}, \ldots, \eta_{r_{n}^{-1}(S), n}\right)$. The partitioning $\eta_{n}$ is complete and sums to unity, although its elements are generically only weakly positive (which leaves a back-door possibility that in some sectors tasks per-

\footnotetext{
${ }^{3}$ An excellent motivation for status-induced occupational sorting can be found in Gola $(2015,2020)$, who builds a two-sector Roy selection model with social status as one of the driving forces of peoples' sorting decisions.

${ }^{4} \mathrm{~A}$ detailed description of the ranking definition can be found in Section 3.
} 
formed by certain occupations might not be necessary at all, which would be equivalent to setting $\left.\gamma_{s, n}=0\right)$.

Assume that after deciding what occupation to pursue, every worker faces a random shock $u_{n}$ drawn from a uniform distribution $\mathcal{U}$ defined on a unit interval. According to its value, the worker is assigned to the sector $s^{*}$ in a way that:

$$
\sum_{r=1, \ldots, r^{*}-1} \eta_{r, n} \leq u_{n} \leq \sum_{r=1, \ldots, r^{*}} \eta_{r, n}
$$

and $s^{*}=r_{n}^{-1}\left(r^{*}\right) .{ }^{5}$ If $u_{n}$ is independently drawn, than the sectoral assignment is random: the wage distributions in all sectors coincide. A perfect positive correlation between $y_{n}$ and $u_{n}$ would imply linear ordering of workers' sorting across sectors. This, however, is not a demanded outcome, as it would imply that sectoral wage distributions are disjoint (have no overlap), which is not observed in the data. In what follows, we propose a given correlation structure between $u_{n}$ and $y_{n}$, which motivates the existence of wage inequality across sectors within the same occupation.

Assume that for a given occupation $n$, the joint distribution of skills after sorting, $G_{n}^{Y}$, and sectoral shocks $U_{n}$ is expressed by a copula function $C_{n}\left(\left(G_{n}^{Y}\right)^{-1}\left(y_{n}\right), u_{n}\right) \cdot{ }^{6} C_{n}$ can take any functional form, and as elaborated in Section 3, we set it to fit the sectoral wage distribution for each $n \in N$. Importantly, a non-degenerated $C_{n}$ determines a sorting rule of workers characterized by $n$-specific skills into sectors. A positive rank correlation between arguments of $C_{n}$ imposes that more skilled (hence high-earning) individuals have a higher chance to be assigned to a more preferred (higher ranked) sector.

Overall, the sector-occupation-specific distribution of wages can be expressed with a use of the occupational distribution: $\left(W_{n}^{-1}\left(F_{s, n}\left(u_{n}\right)\right), F_{s, n}\left(u_{n}\right)\right)$, where the function $F_{s, n}$ aggregates the skill rankings of all individuals, who sort into sector $s: F_{s, n}\left(u_{n}\right)=$ $\int_{0}^{1} \int_{a_{s}}^{b_{s}} C_{n}\left(v_{n}, u_{n}\right) d u_{n} d v_{n}$, recalling that: $r=r_{n}(s), v_{n}=\left(G_{n}^{Y}\right)^{-1}\left(y_{n}\right), a_{s}=\sum_{r^{\prime}=1, \ldots, r-1} \eta_{r^{\prime}, n}$, and $b_{s}=\sum_{r^{\prime}=1, \ldots, r} \eta_{r^{\prime}, n}$. For complex enough copula functions, the sectoral wage distributions have no closed form solutions, therefore we use numerical methods to estimate and depict them.

Equilibrium The labor market equilibrium can be easily articulated as an equalization of demand and supply of efficient labor composites across all occupation-sector cells. Formally:

\footnotetext{
${ }^{5}$ For simplicity, let $\sum_{r \in \emptyset} \eta_{r, n}=0$.

${ }^{6}$ The arguments of the copula function have to be distributed according to a standard uniform, therefore the first argument of $C_{n}$ is filtered by the inverse CDF.
} 
Definition 3. Take any labor marker cell $(s, n)$. For given demand structure $Q_{s}$, prices $P_{s}$, and production technology encompassed in demand for tasks $L_{s, n}$, the labor market is in equilibrium if and only if the wage rates $w_{s, n}$ are such that:

(D4) $L_{s, n}=\int_{F_{s, n}\left(u_{n}\right)} \omega_{n} y_{n}$, for $F_{s, n}\left(u_{n}\right)=[0,1] \times\left[a_{s}, b_{s}\right]$

Finally, the definition of GE is a straightforward conjunction of goods', firms', and labor market equilibria, completed with a macro income consistency criterion.

Definition 4. The economy is in general equilibrium (GE) if and only if: (D1), (D2), (D3), and (D4) hold, and total expenditure equals total income: $Y_{s}=w_{s} L_{s}+i_{s} K_{s}$.

Recalling the properties (I)-(IV) of occupational SUN-type wage distributions, we can add that $(s, n)$-specific wage distributions depend also on complex correlation structure between workers' skills and their choice of sector. In this paper, we focus our attention on a subset of these rich properties of the GE model with occupational sorting. Namely, we exploit properties (I) and (II), as we want to map the medium term implications of the COVID-19 crisis on national labor markets, focusing on the case of Luxembourg. We are mainly interested in sector-specific shocks that represent reductions in demand for consumption goods, selective bankruptcies of firms, and more difficult environment for firm entry. We subsequently quantify their consequences for workers' job opportunities, their susceptibility to unemployment and potential to sort across the labor market, the impact on broad inequality measures, and detailed distributive effects at the level of continuous sector-occupation-specific wage distributions.

\section{Numerical Analysis}

In this section, we explain our numerical strategy in tailoring the model parameters to the data that describe the economy of Luxembourg. The choice of our calibration subject is dictated by the importance of segmentation of its labor market, and polarization of skill requirements across sectors. In brief, some sectors and occupations cluster in terms of task similarity, while workers tend to be strongly specialized in narrow labor market cells. In what follows, we elaborate on our data sources used in the calibration procedure, then we explain our identification assumptions. Finally, we briefly discuss the algorithm of calibration and we illustrate the measures of model fit to the data. 


\subsection{Data}

Labor Market The main data source for the numerical exercise comes from the 2014 Structure of Earnings Survey (SES) by the Eurostat. We dispose of a representative sample of employees from Luxembourg, who are described by a detailed allocation across sectors (NACE 2 digit) and occupations (ISCO 3 digit). Most importantly, the SES provides records of wages in unified units (per hour worked), including yearly bonuses, which crucially simplifies cross-cell comparisons.

We decide to structure our economy into $N+1=5+1$ occupations, and $S=8$ sectors. Table 1 summarizes our aggregation based on ISCO and NACE classifications, respectively. We choose to operate with five market occupations and one non-market occupation, which represents the group of unemployed or inactive workers. Then, we aggregate firms' economic activity into eight sectors. The four largest ones are kept as separate sectors whereas the others are aggregated into bigger categories.

Table 1: The Structure of the Economy: Occupations and Sectors

\begin{tabular}{ccc|ccc}
\hline \hline Occ. Number & Occ. Code & ISCO1d & Sec. Number & Sec. Code & NACE1d \\
\hline 1 & MAN & 1 & 1 & MANU & B, C, D, E \\
2 & PRO & 2,3 & 2 & CONS & F \\
3 & CLC & 4 & 3 & SALE & G \\
4 & SER & 5 & 4 & TRAN & H \\
5 & ELE & $6,7,8,9$ & 5 & LSER & I, N, R, S \\
6 & UNE & $0^{*}$ & 6 & FSER & K \\
& & & 7 & PSER & J, L, M \\
& & & 8 & PUBL & O, P, Q \\
\hline \hline
\end{tabular}

Note: Occupations 1: Managers; 2: Professionals, 3: Clerical Workers, 4: Service and Sale Workers, 5: Less-Skilled and Elementary Occ., 6: Unemployed / Inactive on Labor Market. Sectors: 1: Manufacturing, 2: Construction, 3: Wholesale and Retail Trade, 4: Transport and Storage, 5: Low-Skilled Services, 6: Financial Services, 7: Professional Services, 8: Public Administration, Education, and Health. Source: ISCO, NACE, SES (Eurostat).

With the SES data, we construct non-parametric kernel estimates of occupational wage distributions, as well as sector-occupation-specific kernel densities. ${ }^{7}$ These densities will serve as our reference points for estimating the parameters of the labor market models, as we aim at minimizing distances between empirical and theoretical wage distributions. Moreover, we collect the shares of workers by $(s, n)$-specific labor market shares (which at some point have to be aggregated at the occupational level, including the share of

\footnotetext{
${ }^{7}$ The empirical distributions are arbitrarily censored at 1st and 99th percentile; we also set the smoothing parameter for the Epanechnikov kernel estimation at 2.
} 
unemployed). These values serve as indicators for masses of workers in each labor market cell, and are also fitted in the calibration procedure.

Skill Correlation An important input for our model of labor market sorting is the correlation matrix $\Omega$. Recall that it determines the similarity of tasks across occupations. Thus, its microfoundation origins from skill contents of various occupations, by indicating how easy it is for workers to sort across different job types. Indeed, we calibrate the elements of $\Omega$ using the skill content of tasks data provided by the O*NET database. First, we collect the data on 35 skills used in ISCO 4-digit occupations and we attribute these skills to all workers in the Luxembourgish economy. This gives us a proxy for population-wide distribution of skills at the granularity of 4-digit occupations. Then, we collapse 35 categories into five occupations defined in Table 1. Finally, we compute the correlation between the standardized logarithms of these five vectors, obtaining the matrix presented in Table 2 .

Table 2: The Correlation Matrix of Skills Used in $N$ Occupations

\begin{tabular}{lrrrrr}
\hline \hline & MAN & PRO & CLC & SER & ELE \\
\hline MAN & & & & & \\
PRO & 0.745 & & & & \\
CLC & 0.844 & 0.588 & & & \\
SER & 0.731 & 0.369 & 0.877 & & \\
ELE & -0.726 & -0.966 & -0.538 & -0.298 & \\
\hline \hline
\end{tabular}

As expected, we observe the highest positive relations between Services and Clerical workers. We report the highest negative correlation in occupational skills between Professionals and Elementary Occupations.

Macro Indicators The final inputs for the calibration procedure are the macroeconomic data for Luxembourg. Our main reference in this respect is Eurostat, from which we collect data on stocks of $L_{s}$ and $K_{s}$, as well as the 2018 GDP and its decomposition into employees compensation (determines $1-\theta_{s}$ ) capital compensations ( $\operatorname{sets} \theta_{s}$ ), and corporate profits $\Pi_{s}$ by NACE 1-digit sectors. We also use the data on firms entries and exits by cohorts (i.e. 1Y-5Y survival rates for the last five years, stocks of active enterprises and deaths) to set the values of the probability of bankruptcy, $\rho_{s}$, and the probability of staying on the market $1-G_{s}\left(\varphi_{s}^{*}\right)$. The former is mapped by the fraction of exiting old firms (more than $5 \mathrm{Y}$ of market presence), while the latter is captured by the survival 
rate of young enterprises (below $5 \mathrm{Y}$ of market presence). The total number of enterprises serves as a proxy for the mass of firms, $M_{s}$.

Exogenous Parameters To complete the reference data input for the model, we have to mention three external parameters that we choose to set. The first is the elasticity of substitution between sector-specific goods, $\varepsilon$, which we set equal to four (following a big body of literature in trade and industrial organization and trade, e.g. Simonovska and Waugh, 2014). The same value is ascribed to the elasticity of substitution between goods within each sector, $\sigma$. Third, we assume that the minimal productivity level in each sector $\varphi_{0}$ equals one, which serves as a normalization for nominal values.

\subsection{Identification}

The remaining model objects are determined using model equations. In what follows, we provide a short walk-through of the identification of the macroeconomic environment of the sectoral Melitz (2003) model, and the labor market module.

Sectoral Macroeconomy To identify the sectoral parameters of the Melitz model, we make use of two equilibrium conditions, few model identities, and (crucially) the parametric representation for the distribution of firms' productivities. We dispose of data equivalents of $Y_{s}$ and $\Pi_{s}$, which for a given value of $\sigma$, allows us to derive sector-specific Pareto parameters, $\lambda_{s}$, using equation (19). Taking the number of active firms $M_{s}$ from the data sets the fixed costs of production $f_{s}$, using equation (18). Then, the definition of the probability of staying on the market $1-G_{s}\left(\varphi_{s}^{*}\right)=\left(\varphi_{0} / \varphi_{s}^{*}\right)^{\lambda_{s}}$, for which we also get a moment from the data, sets the value of $\varphi_{s}^{*}$, for a given $\varphi_{0}$. As computed in equation (12), the properties of the Pareto distribution allow us to express the ratio of $\bar{\varphi}_{s}$ to $\varphi_{s}^{*}$ as a function of $\lambda_{s}$ and $\sigma$. Thus, we can compute the model value of $\bar{\varphi}_{s}$. Finally, using the free entry condition (20), we can calibrate the values of the entry costs $\bar{f}_{s}$. As a residual, we set the value for $M_{s}^{e}$, taking all the other inputs in the dynamic equation $\left(\varphi_{0} / \varphi_{s}^{*}\right)^{\lambda_{s}} M_{s}^{e}=\rho_{s} M_{s}$ as given. Our procedure of calibrating the macroeconomic module of the model is feasible as long as the following property holds:

Assumption 1. For each sector $s \in S: \lambda_{s}>\sigma-1$.

Which is fulfilled for every sector $s$ for $\sigma=4$.

Preferences There are two assumption that we have to make in order to achieve identification of preference parameters. The first one imposes that: 
Assumption 2. The sector-specific TFP residuals, $\beta_{s}$, are given.

These values are not identifiable with average productivity levels across sectors, $\bar{\varphi}_{s}$, and indirectly set the overall price level in the economy. Thus, we normalize the value of $P$ :

Assumption 3. The general price index $P$ is equivalent to unity, and is constant across different equilibria,

which technically imposes that all nominal values (e.g. wage rates) are also expressed in real terms whatever counterfactual scenario we consider. Implementing both assumptions, and rearranging equation (4), we can easily compute values of $\alpha_{s}$ for each $s \in S$.

Occupational Cells Within each occupational cell $n \in N$, we observe the empirical distribution of wages that aggregates all workers that decided to sort into job $n$. As in Heckman and Sedlacek (1985), for each $n$ we match four empirical moments that suffice to identify the $n$-specific labor market. These are: $\left(\mathbb{E}\left[W_{n}\right], \mathbb{V}\left[W_{n}\right], \mathbb{S}\left[W_{n}\right], s_{n}\right)$, where $s_{n}=\sum_{s \in S} \eta_{s, n}$. The first three numbers represent the mean, variance and skewness of observed wage distributions in cell $n$. The last moment, $s_{n}$, is the share of all workers that are active in $n$. In what follows, we present the moment matching algorithm that helps to identify the $n$-specific parameters of the SUN distribution. Note that we fit $N=5$ occupational distributions, while we do not observe the $N+1^{\text {st }}$ moments (apart from $\left.s_{N+1}=1-\sum_{n<N} s_{n}\right)$.

Following Arellano-Valle and Azzalini (2006), the moment generating function (mgf) of a $\mathrm{SUN}_{1, N}$ distribution for $n=1, \ldots, N$, including $N+1$ occupations, is given by:

$$
M_{\mathrm{SUN}_{1, N}}\left(t, \Xi_{n}\right)=\exp \left(\xi_{n} t+\omega_{n} t^{2} / 2\right) \Phi_{N}\left(\tau_{n}+\delta_{n} \omega_{n} t ; \Gamma_{n}\right)\left(\Phi_{N}\left(\tau_{n} ; \Gamma_{n}\right)\right)^{-1}
$$

where: $\Phi_{N}\left(\cdot ; \Gamma_{n}\right)$ stays for the cumulative distribution function (CDF) of an $N$-dimensional Normal random variable with mean 0 . Further notational details impose that: $\tau_{n}=$ $\left[\tau_{n-m}\right]_{n}^{T} \in \mathbb{R}^{N}, \delta_{n}=\left[\delta_{n-m}\right]_{n}^{T} \in[-1 ; 1]^{N}$, and the correlation matrix $\Gamma_{n}=\left[\Gamma_{n m p}\right]_{n} \in$ $[-1 ; 1]^{N} \times[-1 ; 1]^{N}$, where, in all cases, for $m, p \in\{1, \ldots, N+1\} /\{n\}$, and $n$ goes through $1, \ldots, N$.

Taking the log of Equation $(30), K(t)=\ln \left(M_{\mathrm{SUN}_{1, N}}\left(t, \Xi_{n}\right)\right)$ we can compute the first three moments of the $\mathrm{SUN}_{1, N}$ distribution for given $\Xi_{n}$ and $N$, and equalize them to observables:

$$
\mathbb{E}\left[W_{i}\right]=\left.K^{\prime}(t)\right|_{t=0} ; \quad \mathbb{V}\left[W_{i}\right]=\left.K^{\prime \prime}(t)\right|_{t=0} ; \quad \mathbb{S}\left[W_{i}\right]=\left.K^{\prime \prime \prime}(t)\right|_{t=0},
$$


where $\left.K^{\prime}(t)\right|_{t=0}=\partial K(t) / \partial t$ evaluated at $t=0$.

The final step includes computing the model shares of workers that sort into given $n=1, \ldots, N+1$. Note that:

$$
s_{n}=\mathbb{P}\left[\forall_{m \neq n} w_{n}>w_{m}+\kappa_{n}\right]=\mathbb{P}\left[\forall_{m \neq n} y_{n-m}+\tau_{n-m}>0\right]=\Phi_{N}\left(\tau_{n} ; \Gamma_{n}\right) .
$$

Overall, for each market occupation $n=1, \ldots, N$ we are left with four identifying equations determining $\left(\mathbb{E}\left[W_{n}\right], \mathbb{V}\left[W_{n}\right], \mathbb{S}\left[W_{n}\right], s_{n}\right)$ and four respective unknowns: $\xi_{n}, \omega_{n}, \omega_{n, N+1}, \kappa_{n}$, where $\omega_{n, N+1}$ is the correlation between the skill used in occupation $n$, and the skill (or preference of remaining) in unemployment (the unobserved $N+1^{\text {st }}$ occupation). The identification of the set of occupational parameters originates from:

Assumption 4. Let $\xi_{N+1}$ be determined by $\mathbb{E}\left[W_{N+1}\right], \omega_{N+1}=1$, and $\kappa_{N+1}=0$.

The parameter $\xi_{N+1}$ is set equal to the empirical moment that represents the logarithm of unemployment benefit in Luxembourg (collected from Eurostat). On the contrary, $\omega_{N+1}$ is normalized to unity, similarly to the cost of leaving the unemployment $\kappa_{N+1}$, which is normalized to zero. There is no condition on the size of the unemployed groups $s_{N+1}$ as it derives as a residual from fitting the shares of workers in all other $N$ market occupations (and is matched to the empirical unemployment rate from the Eurostat). Table 3 summarizes the calibrated values of the SUN distributions of occupational wages.

Table 3: Calibrate Occupational Parameters for SUN Wage Distributions

\begin{tabular}{ccrrrr}
\hline \hline Occ. No. & Occ. Name & \multicolumn{1}{c}{$\xi$} & \multicolumn{1}{c}{$\kappa$} & $\omega$ & $\omega_{, N+1}$ \\
\hline 1 & MAN & 9.83 & 0.16 & 1.03 & 0.02 \\
2 & PRO & 10.58 & 0.34 & 0.66 & 0.26 \\
3 & CLC & 9.86 & -0.20 & 0.69 & -0.12 \\
4 & SER & 9.76 & -0.34 & 0.58 & -0.55 \\
5 & ELE & 9.84 & -0.32 & 0.49 & -0.27 \\
6 & UNE & 9.14 & 0.00 & 1.00 & 1.00 \\
\hline \hline
\end{tabular}

Occupation-Sector Distributions After completing the calibration of occupationspecific wage distributions, we obtain a set of model parameters that determine $N$ SUN distributions. As explained in the previous subsections, we now partition the set of workers in each occupation into $S$ groups that refer to sectoral allocations within each of $N$ market occupations (note that the group of inactive workers in $N+1$ is not allocated across sectors). 
The goal is to find the parameters of occupation-specific copula functions $C_{n}$, that allow us to obtain a decent fit of sectoral wage distributions, for a given sector-occupation shares $\eta_{s, n}$, and given sectoral rankings $r_{n}$. To boost the flexibility of our estimation process, keeping the number of estimated parameters low, we choose to implement a moment fitting algorithm that searches through a family of asymmetric copula functions: $C_{a, b}(u, v)=A\left(u^{a}, v^{b}\right) B\left(u^{1-a}, v^{1-b}\right)$, for any two symmetric copulas $A$ and $B$, and parameters $a, b \in[0,1]$. In particular, we experiment with multiple combinations of $A$ and $B$ originating from standard families (e.g. uniform, Gaussian, Frechet, Gumbel, Frank, Clayton, and FGM copulas), and select the one that allows us to achieve the best fit of occupation-specific collection of $S$ sectoral wage distributions. ${ }^{8}$ Actually, we achieve the best fits with a Frank-Clayton copula, the CDF of which is equal to:

$$
\left.C_{a, b}(u, v)=\ln \left(1 / t_{1}\right) \ln \left(1+\frac{\left(t_{1}^{u^{a}}-1\right)\left(t_{1}^{v^{b}}-1\right)}{t_{1}-1}\right) \cdot\left(\left(u^{1-a}\right)^{-t_{2}}+\left(b^{1-b}\right)^{-t_{2}}-1\right)^{-1 / t_{2}}\right)
$$

with $t_{1}$ and $t_{2}$ representing the standard parameters of Frank and Clayton copulas, respectively. In Table 4 we present the estimated parameter values for each of $N$ copulas.

Table 4: Occupational Copula Functions

\begin{tabular}{clrrcc}
\hline \hline Occ. No. & Occ. Name & \multicolumn{1}{c}{$t_{1}$} & \multicolumn{1}{c}{$t_{2}$} & $a$ & $b$ \\
\hline 1 & MAN & 4.23 & 0.70 & 0.00 & 0.19 \\
2 & PRO & 6.63 & 3.38 & 0.26 & 0.35 \\
3 & CLC & 6.20 & 5.00 & 0.20 & 0.38 \\
4 & SER & 10.02 & 18.03 & 0.26 & 0.58 \\
5 & ELE & 5.49 & 5.42 & 0.46 & 0.11 \\
\hline \hline
\end{tabular}

\subsection{Numerical Algorithms and Model Fit}

The general approach towards calibrating the parameters of labor market relies on moment fitting algorithms with Monte Carlo random search procedures. Essentially, we commence with setting up the goal functions (summarizing Euclidean distances between empirical and model moments, see the previous subsection for the mapping between the two groups), and we aim at finding the vector of model parameters that minimizes it. Unfortunately, in the majority of cases the dimensionality of the parameter space is large,

\footnotetext{
${ }^{8}$ In particular, we aim at minimizing the distance between empirical and model deciles generated by implementing each asymmetric copula. This results in generating $10 \cdot S$ occupational-specific moments, that we fit with four parameters.
} 
thus we proceed with Monte Carlo algorithms to draw new vectors that incrementally improve the fit to the data. The drawing procedures relate strongly to the simulated annealing algorithms that reduce the search space as the number of simulated candidatevectors increases. To support the Monte Carlo algorithm, we complement the search for optimal parameter vectors with two inbuilt $\mathrm{R}$ functions that support local optimization: optim_sa (Flexible Optimization With Simulated Annealing) and the spg function (Spectral Projected Gradient Method for Large-scale Optimization). In conclusion, a variation of the simulated annealing algorithm is used to compute the values of occupational SUN distributions parameters, and to estimate the parameters of asymmetric copula functions in the sector-occupational partition.

Below, we present a series of figures that illustrate the model fit to observed wage distributions for Luxembourg. Figure 1 analyses the whole economy and the following occupational partition. We achieve a very close fit of the aggregated wage distribution, and satisfactory fits of distributions for managers (MAN), clerical workers (CLC), service workers (SER), and people, who work in elementary occupations (ELE). The fit for professional (PRO) is rather imprecise which might be caused by heterogeneity of actual occupations aggregated in this group, and a resulting inadequacy of skill correlations with other occupations.
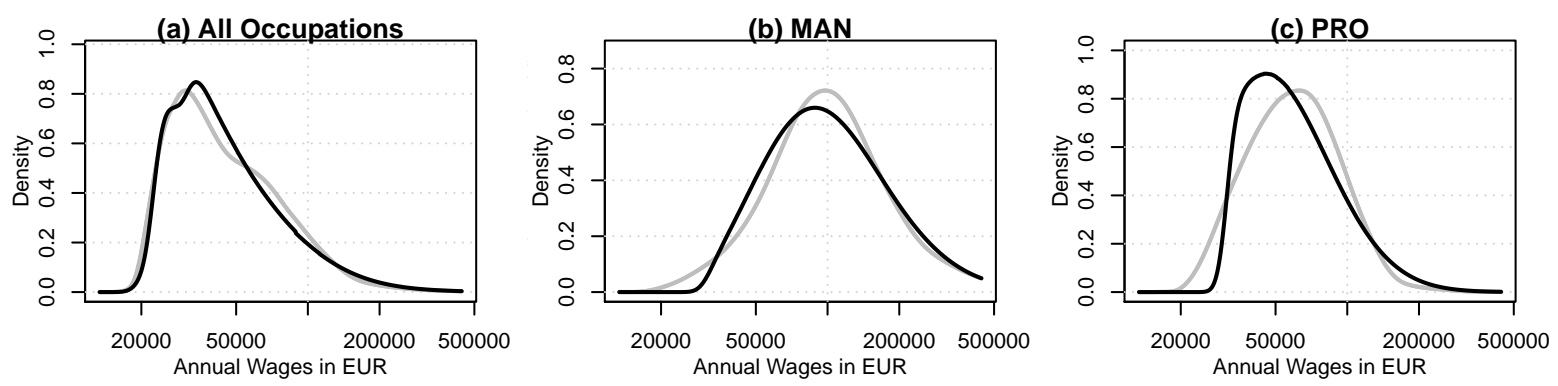

(d) CLC

(e) SER
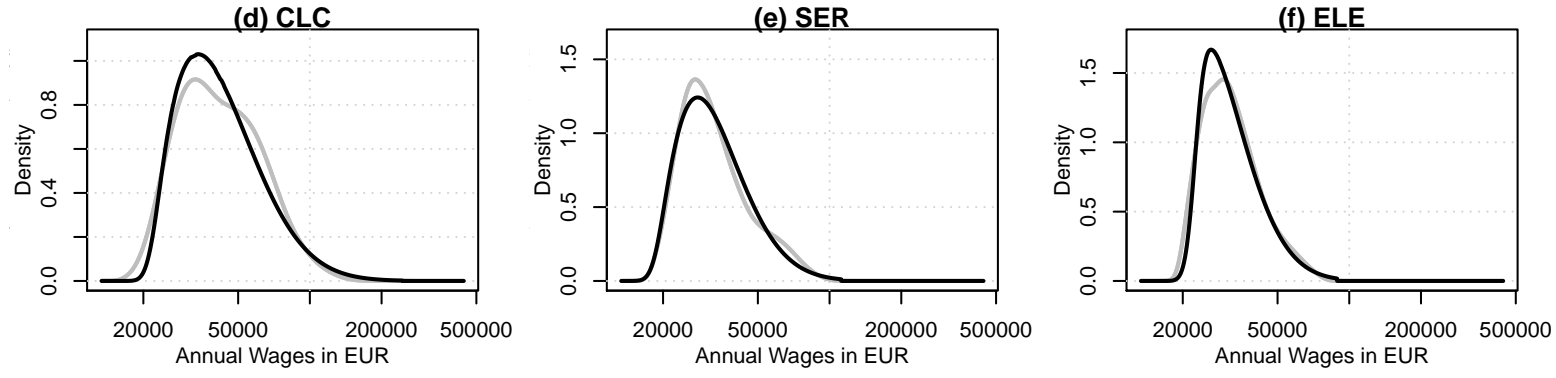

Figure 1: Occupational Wage Fit

Note: Figure 1 illustrates the empirical (in gray) and model (in black) wage distributions for: (a) the whole economy of Luxembourg, (b) managers, (c) professionals, (d) clerical workers, (d) service workers, (e) elementary occupations. 


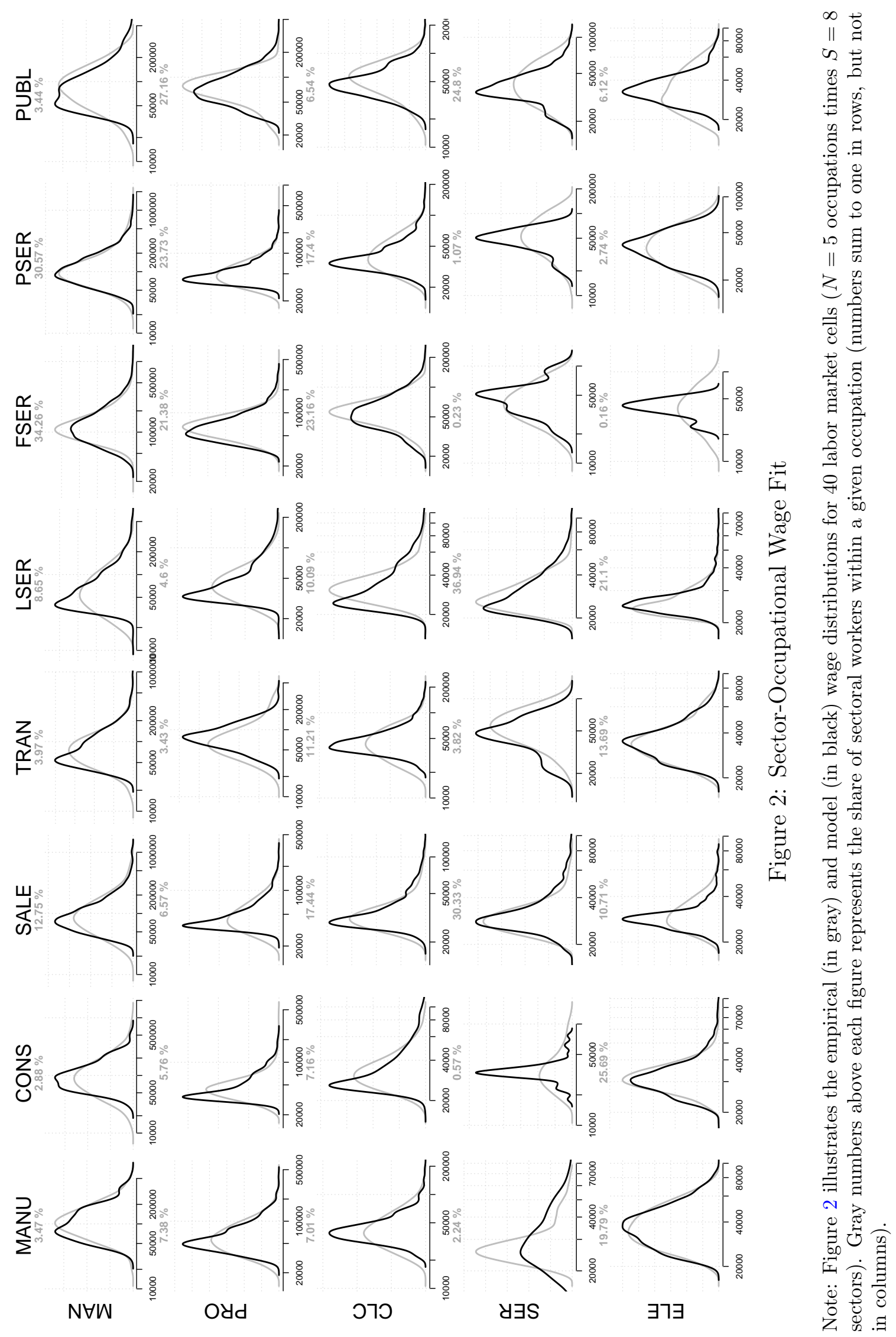


For a detailed illustration of model's fit, Figure 2 presents the sector-occupationspecific wage distributions estimated through workers' partition using occupation-specific rankings of sectors and estimated copula functions. Each occupation (in rows) and sector (in columns) crosses at a unique distribution. Importantly, the fit for most populated occupation-sector cells (indicated by high shares of workers active in it, depicted by gray numbers) is predominantly very good. Some less populated cells, however, are characterized by imprecise estimates, which are driven by low numbers of data points.

With an aim to depict the fitting of occupation-specific copula functions, and their relation to sectoral rankings, Figures 3a-3e illustrate simulated samples of 10.000 individuals in each occupation, and their distribution in the space of sectoral shocks ( $\mathrm{X}$ axis), and skill quantiles ( $\mathrm{Y}$ axis). For each occupation, we also plot the empirical deciles of respective sectoral wage distributions, keeping the size of each sectoral group equal to the length of black lines. The last panel of the Figure summarizes the sectoral rankings for each occupation with 1 representing the least preferred sector and 8 being the most preferred sector. Financial services are most preferred by Managers, Clerical Workers, and Elementary Occupations, while Professionals and Service Workers earn most in public services and transportation sector, respectively.

\section{Simulation Results}

Our quantitative results include two sets of simulations. In the first set, we separately focus on hypothetical shocks for two sectors that have been severely affected by the COVID-19 crisis: sales (SALE) and low-skill services (LSER). We assume that the economy experiences an exogenous drop in the demand for sector specific goods, which is brought about by changes in peoples' preference parameters $\alpha_{s}$. The purpose of these simulations is twofold. First, it shows the macroeconomic consequences of the most probable fallout of the crisis. In this sense, we want to examine the vulnerability of these sectors and the whole economy of Luxembourg to sectoral demand-driven recessions, and the potential consequences for incumbent workers and firms. Second, such an artificial counterfactual enables us to understand the key mechanics of the GE model in a relatively simple and controlled experiment.

In the second set of our results, we implement observed macroeconomic shocks that represent the state of Luxembourgish economy in the second quarter of 2020 (2Q2020 henceforth). This means, we consider a counterfactual state of the world, in which we assume that the initial COVID-19 impact determines a new medium-term equilibrium. We internalize that the impact of pandemic on the macroeconomy is multidimensional 
(a)

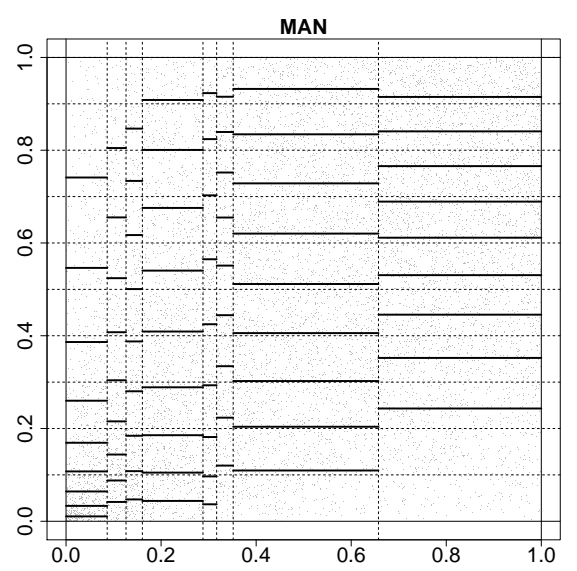

(c)

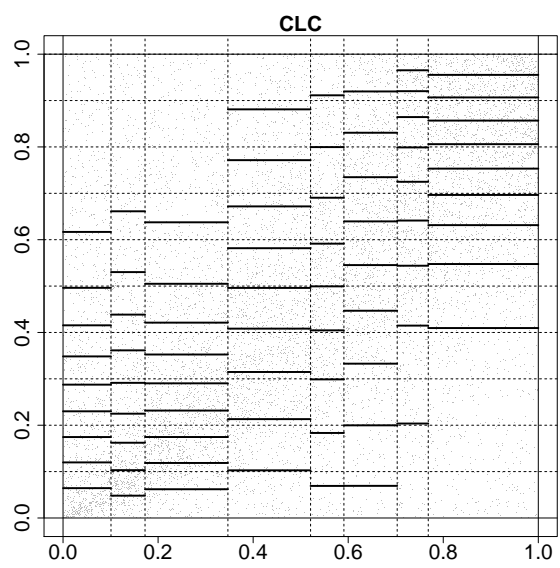

(e)

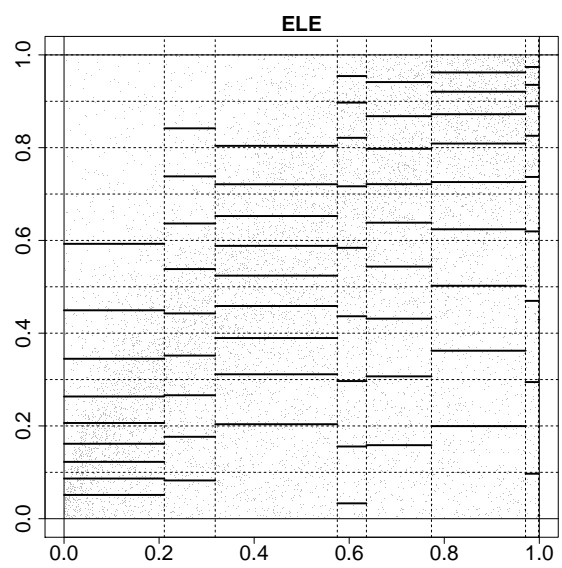

(b)

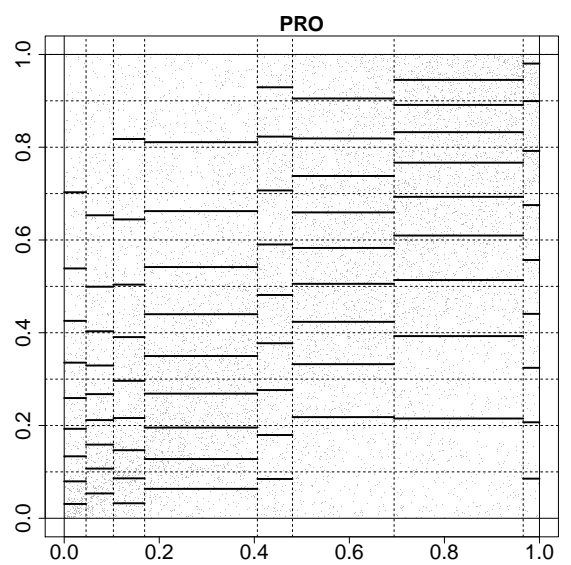

(d)

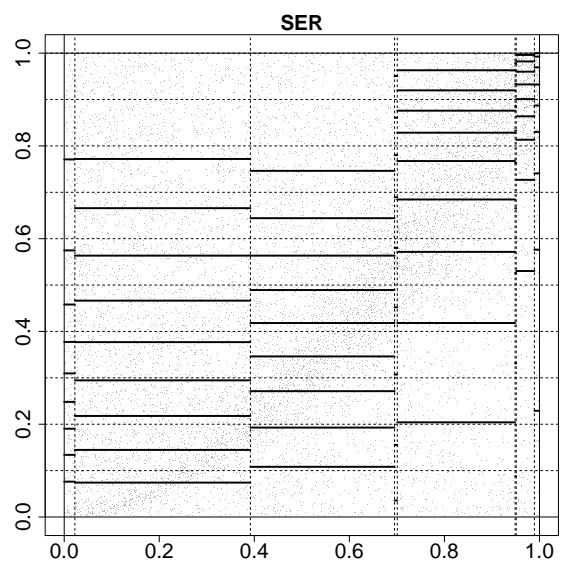

(f)

\begin{tabular}{r|c|c|c|c|c|}
\cline { 2 - 6 } Rank: & MAN & PRO & CLC & SER & ELE \\
\cline { 2 - 6 } $\mathbf{1}$ & LSER & LSER & LSER & MANU & LSER \\
$\mathbf{2}$ & TRAN & CONS & CONS & LSER & SALE \\
$\mathbf{3}$ & PUBL & SALE & SALE & SALE & CONS \\
$\mathbf{4}$ & SALE & PSER & PSER & CONS & PUBL \\
$\mathbf{5}$ & CONS & MANU & MANU & PUBL & TRAN \\
$\mathbf{6}$ & MANU & FSER & TRAN & FSER & MANU \\
$\mathbf{7}$ & PSER & PUBL & PUBL & TRAN & PSER \\
$\mathbf{8}$ & FSER & TRAN & FSER & PSER & FSER \\
\hline
\end{tabular}

Figure 3: Estimated Copula Functions and Sectoral Rankings

Note: Figures 3a-3e depicts simulations from five occupational copulas (gray points) and compares them to empirical deciles of sectoral wage distributions (black lines). The lengths of lines correspond to the shares of sectoral employment within occupations. Y axes represent skill rankings, while $\mathrm{X}$ axes depict sector rankings. Figure 3f presents the rankings of sectors for five occupations from the lowest rank (1) to the highest rank (8). 
and complex. On the one hand, COVID-19 has caused heterogeneous shifts in demand across product types. The extreme examples might include unprecedented fall in demand for restaurants, hotels and travel services due to the lockdown imposed in Luxembourg (arguably backed by peoples' diminished willingness to consume these services in times of pandemic), with a simultaneous rise in the demand for basic food products. In the medium run, such a shock affects not only quantities sold, but also product prices, thus indirectly impacts firms' profitability. On the other hand, due to COVID-19 firms' ability to operate per se has been seriously challenged. Employers have become more vulnerable to bankruptcies and must suffer higher non-labor costs for their day-to-day activity. In technical terms this means that apart from sector-specific changes in parameters $\alpha_{s}$, we also consider variations in the probability of bankruptcy $\rho_{s}$, and the fixed costs $f_{s}$. We solve for a new medium-term equilibrium of the model endogenizing the adjustment mechanisms across all markets, but keeping the market price of capital constant (forcing equilibrium capital adjustments).

In Table 5, we summarize the magnitudes of respective shocks for all scenarios considered. Note that simulations $1 \mathrm{a}$ and $1 \mathrm{~b}$ consider hypothetical changes in selected preference parameters, while the magnitudes in the simulation 2 are determined by observations, and explained in subsection 4.2 .

Table 5: Demand Shocks Due to COVID-19

\begin{tabular}{lcccccccc}
\hline \hline \multicolumn{2}{l}{ Scenario 1a (focus on LSER): } & & & & & \\
Var. & MANU & CONS & SALE & TRAN & LSER & FSER & PSER & PUBL \\
\hline$d Q$ & $0.0 \%$ & $0.0 \%$ & $0.0 \%$ & $0.0 \%$ & $-50.0 \%$ & $0.0 \%$ & $0.0 \%$ & $0.0 \%$ \\
$d \alpha$ & $0.0 \%$ & $0.0 \%$ & $0.0 \%$ & $0.0 \%$ & $-13.0 \%$ & $0.0 \%$ & $0.0 \%$ & $0.0 \%$ \\
\hline
\end{tabular}

Scenario $1 b$ (focus on SALE):

\begin{tabular}{ccccccccc} 
Var. & MANU & CONS & SALE & TRAN & LSER & FSER & PSER & PUBL \\
\hline$d Q$ & $0.0 \%$ & $0.0 \%$ & $-50.0 \%$ & $0.0 \%$ & $0.0 \%$ & $0.0 \%$ & $0.0 \%$ & $0.0 \%$ \\
$d \alpha$ & $0.0 \%$ & $0.0 \%$ & $-13.0 \%$ & $0.0 \%$ & $0.0 \%$ & $0.0 \%$ & $0.0 \%$ & $0.0 \%$ \\
\hline
\end{tabular}

Scenario 2 (2Q2020):

\begin{tabular}{ccccccccc} 
Var. & MANU & CONS & SALE & TRAN & LSER & FSER & PSER & PUBL \\
\hline$d Q$ & $-12.8 \%$ & $-11.3 \%$ & $-26.6 \%$ & $33.1 \%$ & $-21.6 \%$ & $-5.3 \%$ & $11.5 \%$ & $4.9 \%$ \\
$d \alpha$ & $-2.7 \%$ & $-2.4 \%$ & $-6.0 \%$ & $5.9 \%$ & $-4.7 \%$ & $-1.1 \%$ & $2.2 \%$ & $1.0 \%$ \\
$d \rho$ & $17.8 \%$ & $15.4 \%$ & $30.1 \%$ & $-34.1 \%$ & $18.1 \%$ & $3.0 \%$ & $-11.7 \%$ & $7.8 \%$ \\
$d f$ & $4.5 \%$ & $5.6 \%$ & $5.3 \%$ & $10.6 \%$ & $9.4 \%$ & $4.2 \%$ & $7.8 \%$ & $7.8 \%$ \\
\hline \hline
\end{tabular}

Source: Eurostat, Editus, Chambre de Commerce and own calculations. $Q$ stands for real demand, $\alpha$ are the sector-specific demand parameters, $\rho$ is the probability of bankruptcy, while $f$ is the fixed cost. 


\subsection{Scenario 1: Sectoral Experiments for LSER and SALE}

Controlled, unisectoral shocks provide a straightforward illustration of the model mechanics. According to Table 5, in both scenarios we implemented exogenous $50 \%$ decreases in real consumption, which correspond to reductions in preference parameters $\alpha_{s}$ of approximately 13\%. ${ }^{9}$ Figures $4 \mathrm{a}$ and $5 \mathrm{a}$ (for LSER and SALE scenario, respectively) depict the main aggregates that evolve endogenously in our GE system. Panels (i) focus on macroeconomic indicators: changes in GDP, price indexes, real demand, efficient labor composite employed, and the mass of firms operating in each of eight sectors. Imposing a strong negative demand shock in LSER and SALE has detrimental consequences for the value added, which drops by $29 \%$ and $27 \%$, respectively. In both scenarios prices and quantities plunge, and the employment of efficient labor composite decreases. Importantly, LSER and SALE sectors are strongly interrelated, as the labor market spillovers between the two are strong. In the LSER simulation, SALE employment is strongly negatively affected, and vice versa for the SALE simulation. This relationship roots in the calibrated production technology, which grounds on a similar task composition: intensity in SER occupations. This feature is visible in panels (ii) of Figures $4 \mathrm{a}$ and $5 \mathrm{a}$, which depicts changes in the efficient labor employed disaggregated at the sectoral and occupational level. Service occupations are predominantly negatively affected in both instances (ELE occupations being the second group most affected in the LSER scenario, while CLC are hit in the SALE scenario). Interestingly, professionals (PRO occupation) gain the most in both simulations, as they are demanded by those sectors that are less affected by demand shocks, e.g. FSER and PSER.

Panels (iii) and (iv) illustrate the selection of workers that end up in specific labor market cells. The average level of skills and the number of employed people tend to move in opposite directions. Outflows of workers lead to improvements in average skills, as those who quit are negatively selected. This takes place for the SER and ELE jobs in scenario LSER, and SER and CLC jobs in the SALE scenario. Note that in the latter case, we also record a significant selection effect at the sectoral level: despite the negative shock and a drop in the efficient labor composite, the number of individual workers increases in SALE, but their selection is significantly worse. The explanation for this phenomenon relates to the sectoral rankings which determine workers' assignment within occupations. A drop in demand in SALE puts this sector at the bottom of rankings in all occupations except MAN, which imposes that workers of the least skill level transfer

\footnotetext{
${ }^{9}$ Note that the resulting changes in $\alpha_{s}$ equal to $\left(d Q_{s} / Q_{s}\right)^{1 / \varepsilon}$, according to equation (4), which assumes that sectoral price levels have not adjusted. This assumption is not that restrictive considering the speed of the shock and low chances of achieving the medium-term equilibrium within few weeks after it.
} 
to SALE. This significantly deteriorates the selection of incumbents in SALE, while the new inflows originate from CONS, LSER, and also PUBL sectors. Note that a similar phenomenon is not present in the LSER simulation: only because LSER occupies the last ranking position in four out of five occupations already before the negative demand shock. Thus, the sector-specific selection channel is absent in panels (iii) and (iv) of Figure 4a, and only occupation-specific selection operates. Overall, both shocks have devastating consequences for the Luxembourgish economy, with country-wide GDP falling by $4.1 \%$ and $5.0 \%$, number of firms falling by $7.1 \%$ and $7.3 \%$, while unemployment rate surging by $27 \%$ and $21 \%$ in two respective scenarios. ${ }^{10}$

Figures $4 \mathrm{~b}$ and $5 \mathrm{~b}$ illustrate changes in wage distributions in the whole Luxembourgish economy, and at sectoral levels, for LSER and SALE scenarios, respectively. Lower demand for LSER services reduces the quantiles of respective wage distributions by 4 to $6 \%$ in the majority of sectors, LSER being hit most severely. What needs to be underlined, is a persistent and common dichotomy of losses between left-tail and right-tail quantiles. Inequality in all sectors, and in the aggregated economy, increases as lower parts of wage distributions lose more than the upper segments. In the SALE scenario the general distributive effect is slightly different. First, according to Figure 5b, all quantiles of the economy-wide wage distribution lose approximately $4 \%$ to $5 \%$. Second, we see a huge drop in wages payed to SALE workers: up to over $15 \%$ for the 30 th percentile. Third, there is a significant gain in wages payed in LSER sector, as it visibly "benefits" from the improved selection of less-skilled workers (a similar mechanism engaging ex-SALE workers from PRO and CLC occupations can be observed in the right tail of the CONS sector wage distribution). Thus, the importance of inter-sectoral self-selection of workers strongly affects the macroeconomic outcomes, as the workers and firms active in a sector in recession lose in two ways. The initial drop in value added and wages motivates more skilled workers to leave, which depreciates the sectoral productivity even more. Those more productive workers execute their outside option and choose to move to a sector that is less violently hit by the shock, without altering their occupational tasks. The missing vacancies are filled by less skilled individuals. Apparently, the LSER sector reaps the majority of these gains, as it overtakes SALE in four occupational rankings and accumulates more productive ex-SALE workers.

The last comment touches the issue of inter-occupational mobility of workers. In both

\footnotetext{
${ }^{10}$ Note that the change in the mass of firms is identical to the change in GDP, as predicted by equation (18). This is a feature of the consumption shock and holds as long as $f_{s}$ are kept constant. Note also that the cutoff and average productivities do not change in consumption scenarios as well as $\bar{f}_{s}$ and $\delta_{s}$ do not change. Both implication will not hold in the next scenario, in which we vary the above mentioned parameters.
} 
Figure 4: Scenario 1a: 50\% Reduction in Real Demand in LSER

(a) Macroeconomic and Labor Market Aggregates

(i) Macro indicators

\begin{tabular}{rrrrrr} 
& Y & P & Q & L & M \\
\cline { 2 - 6 } MANU & $-3,00 \%$ & $-0,37 \%$ & $-2,64 \%$ & $-1,08 \%$ & $-3,00 \%$ \\
\cline { 2 - 6 } CONS & $-2,93 \%$ & $-0,40 \%$ & $-2,54 \%$ & $-1,44 \%$ & $-2,93 \%$ \\
\cline { 2 - 6 } SALE & $-3,42 \%$ & $-0,23 \%$ & $-3,20 \%$ & $-1,85 \%$ & $-3,42 \%$ \\
\cline { 2 - 6 } TRAN & $-3,29 \%$ & $-0,28 \%$ & $-3,02 \%$ & $-1,43 \%$ & $-3,29 \%$ \\
LSER & $-28,75 \%$ & $-12,36 \%$ & $-18,70 \%$ & $-2,68 \%$ & $-28,8 \%$ \\
FSER & $-1,36 \%$ & $-0,93 \%$ & $-0,43 \%$ & $0,13 \%$ & $-1,36 \%$ \\
PSER & $-2,23 \%$ & $-0,64 \%$ & $-1,60 \%$ & $-0,03 \%$ & $-2,23 \%$ \\
PUBL & $-2,18 \%$ & $-0,65 \%$ & $-1,54 \%$ & $-0,57 \%$ & $-2,18 \%$ \\
Aggr. & $-4,09 \%$ & - & $-2,05 \%$ & $-0,80 \%$ & $-7,09 \%$ \\
\hline
\end{tabular}

(iii) Average Skill Level

\begin{tabular}{rlllrl} 
& MAN & PRO & CLC & SER & ELE \\
\hline MANU & $-0,61 \%$ & $-0,82 \%$ & $-0,40 \%$ & $-6,81 \%$ & $1,14 \%$ \\
\cline { 2 - 5 } CONS & $-1,82 \%$ & $-1,09 \%$ & $-0,69 \%$ & $1,56 \%$ & $1,07 \%$ \\
SALE & $-0,11 \%$ & $-0,68 \%$ & $-0,42 \%$ & $1,00 \%$ & $1,09 \%$ \\
\cline { 2 - 5 } TRAN & $-0,28 \%$ & $-0,57 \%$ & $-0,54 \%$ & $1,08 \%$ & $1,10 \%$ \\
LSER & $-0,47 \%$ & $-0,90 \%$ & $-0,46 \%$ & $1,59 \%$ & $1,14 \%$ \\
FSER & $-0,12 \%$ & $-0,52 \%$ & $-0,30 \%$ & $1,17 \%$ & $0,95 \%$ \\
PSER & $-0,14 \%$ & $-0,77 \%$ & $-0,52 \%$ & $0,93 \%$ & $1,06 \%$ \\
PUBL & $0,93 \%$ & $-0,45 \%$ & $-0,24 \%$ & $0,98 \%$ & $1,06 \%$ \\
\hline
\end{tabular}

(ii) Total Efficient Composite

\begin{tabular}{llllll}
\hline MAN & PRO & CLC & SER & ELE & UNE \\
\hline
\end{tabular}

\begin{tabular}{llllll} 
MANU & $-0,19 \%$ & $0,46 \%$ & $-0,53 \%$ & $-6,72 \%$ & $-2,19 \%$ \\
\hline
\end{tabular}

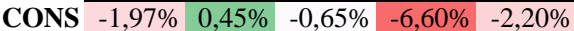

\begin{tabular}{llllll} 
SALE & $-0,12 \%$ & $0,70 \%$ & $-0,48 \%$ & $-6,65 \%$ & $-2,20 \%$ \\
\hline
\end{tabular}

\begin{tabular}{llllll} 
TRAN & $-0,28 \%$ & $0,41 \%$ & $-0,54 \%$ & $-6,63 \%$ & $-2,20 \%$ \\
\hline
\end{tabular}

\begin{tabular}{llllll} 
LSER & $-0,30 \%$ & $0,43 \%$ & $-0,45 \%$ & $-6,63 \%$ & $-2,19 \%$ \\
\hline
\end{tabular}

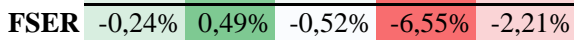

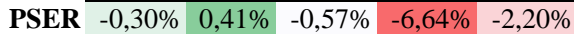

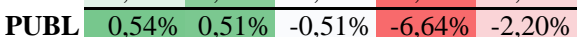

\begin{tabular}{lllllll} 
Aggr. & $-0,08 \%$ & $1,13 \%$ & $-0,09 \%$ & $-7,59 \%$ & $-3,26 \%$ & $26,5 \%$ \\
\hline
\end{tabular}

(iv) Number of Employees

\begin{tabular}{lllll}
\hline MAN & PRO & CLC & SER & ELE \\
\hline
\end{tabular}

\begin{tabular}{lllllll} 
MANU & $2,86 \%$ & $2,24 \%$ & $1,87 \%$ & $1,76 \%$ & $-1,31 \%$ \\
\hline
\end{tabular}

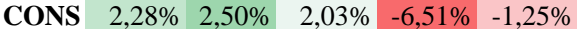

\begin{tabular}{lllllll} 
SALE & $2,41 \%$ & $2,33 \%$ & $1,94 \%$ & $-6,04 \%$ & $-1,27 \%$ \\
\hline
\end{tabular}

\begin{tabular}{llllll} 
TRAN & $2,43 \%$ & $1,93 \%$ & $1,99 \%$ & $-6,09 \%$ & $-1,28 \%$ \\
\hline
\end{tabular}

\begin{tabular}{llllll} 
LSER & $2,60 \%$ & $2,29 \%$ & $2,00 \%$ & $-6,57 \%$ & $-1,32 \%$ \\
\hline
\end{tabular}

\begin{tabular}{llllll} 
FSER & $2,30 \%$ & $1,96 \%$ & $1,77 \%$ & $-6,10 \%$ & $-1,15 \%$ \\
\hline
\end{tabular}

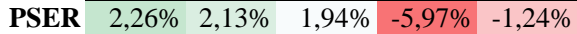

\begin{tabular}{llllll} 
PUBL & $2,04 \%$ & $1,91 \%$ & $1,72 \%$ & $-6,01 \%$ & $-1,24 \%$ \\
\hline
\end{tabular}

(b) Changes in Aggregated Wage Distributions
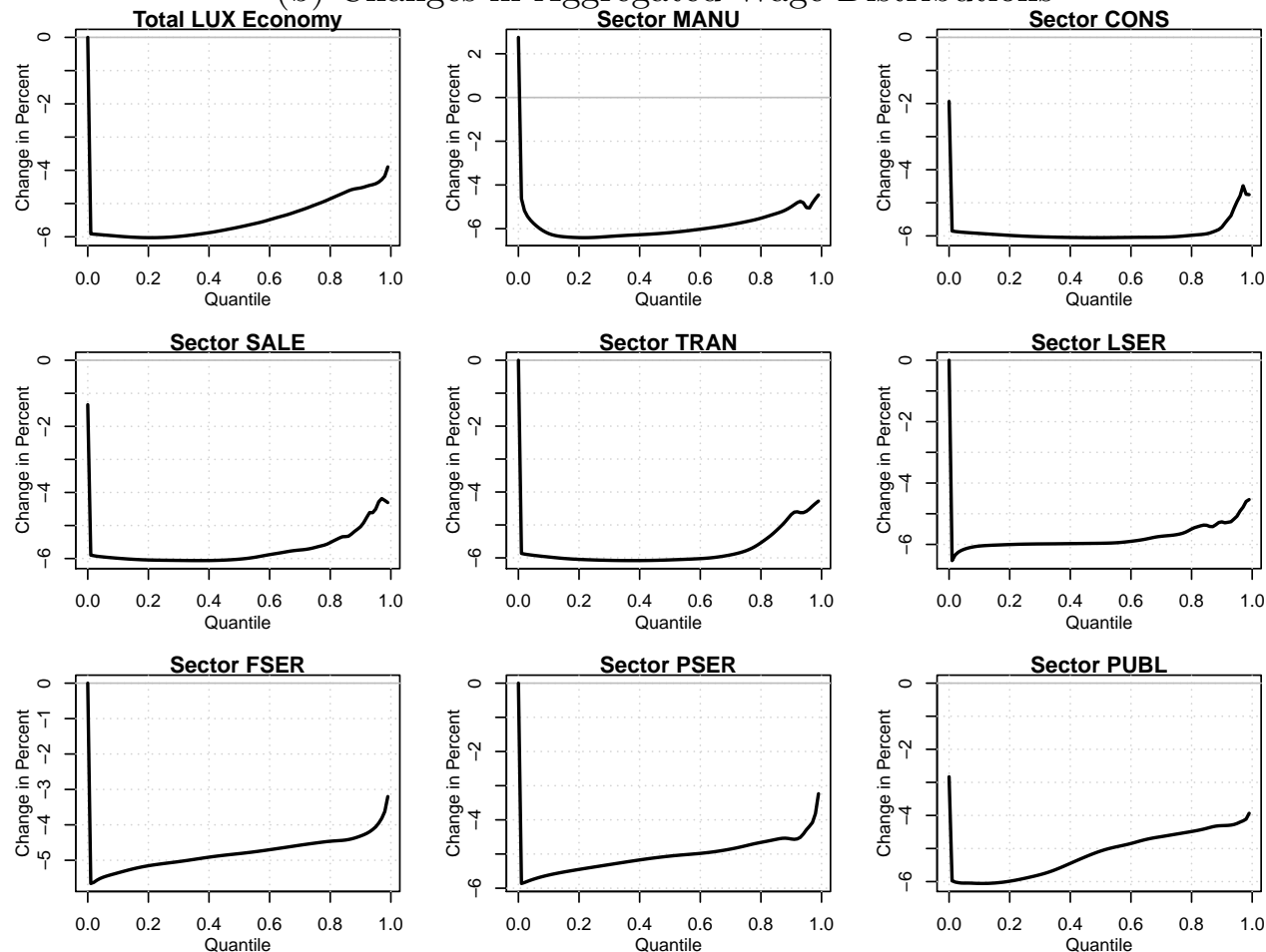

Note: Figures 4a reports changes in (i) key macroeconomic indicators (GDP, price indexes, real production, total labor composite, and the mass of firms), (ii) total efficient labor composites, (iii) average skill levels, and (iv) numbers of employees by $(s, n)$ labor market cells. Figure $4 \mathrm{~b}$ illustrates relative changes in wage rates for quantiles of respective wage distributions by sectors. 
Figure 5: Scenario 1b: 50\% Reduction in Real Demand in SALE

(a) Macroeconomic and Labor Market Aggregates

(i) Macro indicators

\begin{tabular}{|c|c|c|c|c|c|}
\hline & $\mathbf{Y}$ & $\mathbf{P}$ & $\bar{Q}$ & $\mathbf{L}$ & $\bar{M}$ \\
\hline ANU & $-2,84 \%$ & $-0,75 \%$ & $-2,11 \%$ & $-0,46 \%$ & $-2,84 \%$ \\
\hline $\mathrm{CON}$ & $-2,13 \%$ & $-1,00 \%$ & $-1,14 \%$ & $-0,28 \%$ & $-2,13 \%$ \\
\hline SALE & $-26,71 \%$ & $-13,47 \%$ & $-15,3 \%$ & $-2,15 \%$ & $-26,7 \%$ \\
\hline TRAN & $-3,00 \%$ & $-0,70 \%$ & $-2,31 \%$ & $-0,70 \%$ & $-3,00 \%$ \\
\hline LSER & $-3,43 \%$ & $-0,56 \%$ & $-2,89 \%$ & $-1,29 \%$ & $-3,4 \%$ \\
\hline FSER & $-2,30 \%$ & $-0,94 \%$ & $-1,37 \%$ & $-0,47 \%$ & $-2,30 \%$ \\
\hline SER & $-3,13 \%$ & $-0,66 \%$ & $-2,49 \%$ & $-0,43 \%$ & $-3,13 \%$ \\
\hline PUBL & $-2,56 \%$ & $-0,85 \%$ & $-1,73 \%$ & $-0,58 \%$ & $-2,56 \%$ \\
\hline 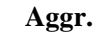 & $-5,02 \%$ & - & $-2,58 \%$ & $-0,66 \%$ & $-7,31 \%$ \\
\hline
\end{tabular}

(ii) Total Efficient Composite

(iii) Average Skill Level

\begin{tabular}{lrrrrr}
\cline { 2 - 6 } & \multicolumn{1}{c}{ MAN } & PRO & CLC & \multicolumn{1}{c}{ SER } & ELE \\
\hline MANU & $0,12 \%$ & $-0,07 \%$ & $0,50 \%$ & $-3,10 \%$ & $0,42 \%$ \\
\cline { 2 - 6 } CONS & $0,53 \%$ & $4,75 \%$ & $8,10 \%$ & $1,78 \%$ & $0,32 \%$ \\
SALE & $-9,20 \%$ & $-4,82 \%$ & $-4,38 \%$ & $-2,41 \%$ & $-11,8 \%$ \\
\cline { 2 - 6 } TRAN & $23,78 \%$ & $0,02 \%$ & $0,53 \%$ & $1,23 \%$ & $0,34 \%$ \\
LSER & $0,29 \%$ & $1,08 \%$ & $4,85 \%$ & $4,79 \%$ & $7,41 \%$ \\
\cline { 2 - 5 } FSER & $0,35 \%$ & $-0,05 \%$ & $0,48 \%$ & $1,32 \%$ & $0,28 \%$ \\
PSER & $0,29 \%$ & $0,03 \%$ & $0,50 \%$ & $1,10 \%$ & $0,32 \%$ \\
\hline PUBL & $21,13 \%$ & $0,08 \%$ & $0,32 \%$ & $1,17 \%$ & $0,28 \%$ \\
\hline \hline
\end{tabular}

\begin{tabular}{llllll}
\hline MAN & PRO & CLC & SER & ELE & UNE
\end{tabular}

\begin{tabular}{llllll} 
MANU & $-0,85 \%$ & $0,01 \%$ & $-1,99 \%$ & $-5,33 \%$ & $-0,47 \%$ \\
\hline
\end{tabular}

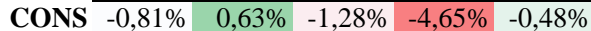

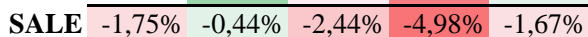

\begin{tabular}{lrrrrr} 
TRAN & $1,44 \%$ & $0,05 \%$ & $-1,98 \%$ & $-4,69 \%$ & $-0,51 \%$ \\
\hline
\end{tabular}

\begin{tabular}{llllll} 
LSER & $-0,84 \%$ & $0,01 \%$ & $-1,58 \%$ & $-4,40 \%$ & $0,22 \%$ \\
\hline
\end{tabular}

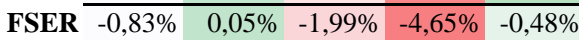

\begin{tabular}{llllll}
\hline PSER & $-0,83 \%$ & $0,05 \%$ & $-1,98 \%$ & $-4,70 \%$ & $-0,48 \%$ \\
\hline
\end{tabular}

PUBL \begin{tabular}{llllll}
$1,15 \%$ & $0,06 \%$ & $-2,00 \%$ & $-4,70 \%$ & $-0,38 \%$ \\
\hline
\end{tabular}

\begin{tabular}{lllllll} 
Aggr. & $-1,15 \%$ & $0,05 \%$ & $-2,49 \%$ & $-5,86 \%$ & $-0,74 \%$ & $20,7 \%$ \\
\hline
\end{tabular}

(iv) Number of Employees

\begin{tabular}{rrrrrr}
\cline { 2 - 6 } & MAN & PRO & \multicolumn{1}{c}{ CLC } & \multicolumn{1}{c}{ SER } & ELE \\
\cline { 2 - 6 } MANU & $1,09 \%$ & $0,66 \%$ & $-0,87 \%$ & $-1,00 \%$ & $0,79 \%$ \\
\cline { 2 - 6 } CONS & $0,72 \%$ & $-3,38 \%$ & $-7,18 \%$ & $-5,06 \%$ & $0,87 \%$ \\
SALE & $10,5 \%$ & $5,20 \%$ & $3,70 \%$ & $-1,33 \%$ & $13,4 \%$ \\
\hline TRAN & $-16,3 \%$ & $0,60 \%$ & $-0,90 \%$ & $-4,58 \%$ & $0,82 \%$ \\
LSER & $0,94 \%$ & $-0,49 \%$ & $-4,59 \%$ & $-7,54 \%$ & $-5,12 \%$ \\
FSER & $0,89 \%$ & $0,68 \%$ & $-0,86 \%$ & $-4,62 \%$ & $0,91 \%$ \\
\hline PSER & $0,94 \%$ & $0,60 \%$ & $-0,88 \%$ & $-4,47 \%$ & $0,87 \%$ \\
PUBL & $-14,8 \%$ & $0,56 \%$ & $-0,72 \%$ & $-4,53 \%$ & $1,01 \%$ \\
\hline \hline
\end{tabular}

(b) Changes in Aggregated Wage Distributions
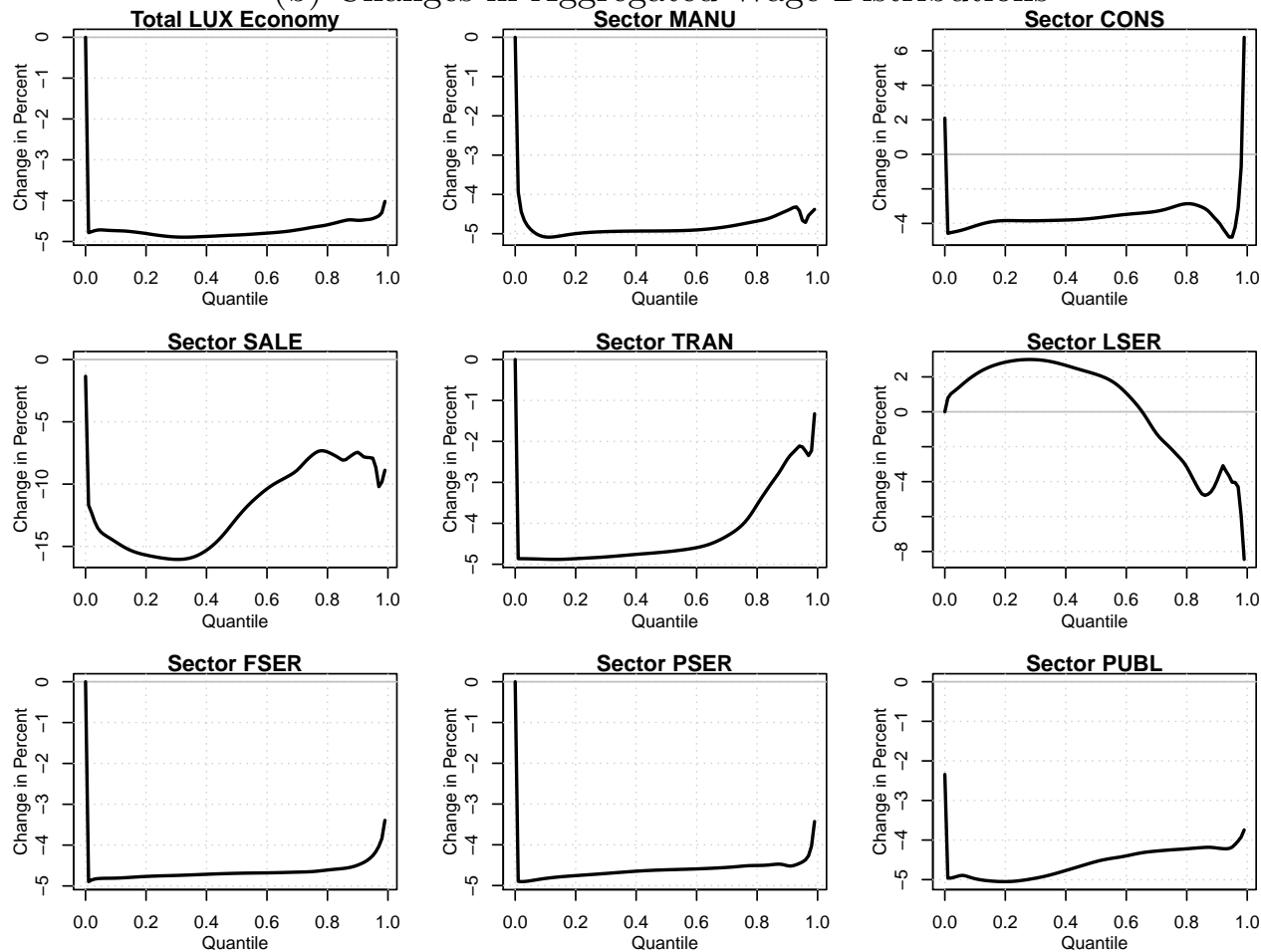

Note: Figures 5a reports changes in (i) key macroeconomic indicators (GDP, price indexes, real production, total labor composite, and the mass of firms), (ii) total efficient labor composites, (iii) average skill levels, and (iv) numbers of employees by $(s, n)$ labor market cells. Figure $5 \mathrm{~b}$ illustrates relative changes in wage rates for quantiles of respective wage distributions by sectors. 
scenarios, the model predicts moderate flows between market occupations: the predominant flows are directed into unemployment. However, as panel (ii) of Figure 4a depicts, the unconditional shares of efficient labor (reported in the last row labeled Aggr.), there is an increase in the workforce in PRO occupations, which could not be achieved without inter-occupational mobility (mainly from MAN and CLC occupations). However, looking at absolute numbers, the drops in MAN and CLC cannot compensate the increases in PRO workers, which indicates that some blue-collar workers from SER and ELE occupations actually upgraded into management or office jobs. In the SALE scenario the picture is less optimistic, with majority of workers moving down the ladder of occupational hierarchy: either choosing lower paid jobs, or moving into unemployment.

\subsection{Scenario 2: Economic Effects of COVID-19}

To set up the 2Q2020 COVID-19 scenario we use the data provided by Eurostat on the changes in total GDP by NACE 1-digit sector. We aggregate it into $S$-sectoral weighted averages, and we compute the respective magnitudes of changes in real consumption $Q_{s}$, which proxies for the initial demand shocks that sets the new values of $\alpha_{s}$. The third panel of Table 5 summarizes the inputs our data-driven scenario in the first line, and the respective outputs for changes in preference parameters in the second line. Indeed, SALE and LSER sectors experience the most severe negative shocks $-27 \%$ and $-22 \%$ respectively, followed by MANU and CONS that lose around 12\%. Surprisingly, TRAN and PSER sectors gain substantially with the 2Q2020 GDP increases by $33 \%$ and $12 \%$ respectively. Note that we treat these changes in GDP as permanent demand shocks, thus they define the new structure of consumption in the medium-term equilibrium.

Then, we use two additional data sources to translate these demand shocks into changes in firm environment. First, we use the Editus data to compute the new values of sectoral default probabilities, $\rho_{s}$. Editus provides data on balance sheets and profit and loss accounts for the universe of Luxembourgish firms. We relate the risk of bankruptcy to the liquidity risk, and we proxy it with a synthetic binary indicator that keeps track of values of current and quick liquidity ratios. ${ }^{11}$ Thus a proportionate increase in the share of firms that fall outside the acceptance set in terms of liquidity indicators (brought about by firm-by-firm computation of reductions in the demand for goods, implying lower current assets and higher current liabilities), increases the sectoral risk of bankruptcy in our model, see the third line in the third panel of Table 5. Second, we use the survey

\footnotetext{
${ }^{11}$ Current liquidity ratio equals current assets over short-term liabilities. Quick liquidity ratio equals cash and other liquid assets over short-term liabilities. We assume that the first ratio should be above 1.5, whereas the second one: above 0.9 .
} 
data provided by the Chambre de Commerce in Luxembourg, in which entrepreneurs are asked about the perceived increase in running costs due to COVID-19. Computing sectoral aggregates leads us to the spikes in fixed costs of production as indicated in the last line of Table 5.

Given the distribution of the COVID-19 economic shock, our model is expected to reveal visible sectoral and occupational heterogeneity in the GE adjustment mechanism. The mechanism that originates from the goods' demand is identical to what is analyzed in the first set of results: lower sectoral demand reduces sectoral prices, wages, labor demands and causes firms to exit the sector. Simultaneously, it imposes occupational and sectoral reshuffling of workers: the selection of workers in "green" sectors improves, while in "red" sectors they are negatively selected. Moreover, occupations which are intensively used in "red" sectors experience visible outflows of workers. The further mechanism in the 2Q2020 simulation deteriorates the business environment, and affects the quantity and the productivity of firms active across sectors. Higher default probability unambiguously reduces the expected discounted firm value, and discourages firms from entering the market. Less entries increase the probability of staying and deteriorate the productivity of stayers. Higher fixed costs, on the contrary, diminish the equilibrium mass of firms though the zero profit condition, but improve the selection of stayers, as the cutoff revenue increases. The interplay between the two forces has a direct impact on equilibrium values of $M_{s}$ and $\varphi_{s}^{*}$ that determine the new distribution of firm productivities.

Figure 6a panel (i) summarizes the macroeconomic consequences of the imposed demand shock. The model predicts that in the medium-term GE reductions in sectoral GDPs for SALE and LSER sectors reach the level of approximately $-20 \%$, while MANU and CONS experience reductions between $-10 \%$ and $-15 \%$. TRAN and PSER sectors grow due to COVID-19 by $13 \%$ and $8 \%$ respectively. The initial change in real production is mitigated by a shift in prices of sectoral goods, which (in the majority of cases) constitutes around $15-30 \%$ of the total adjustment in GDP. The number of active firms no longer follows changes in GDP, and moves drastically down in SALE and LSER (over -25\%), with only slightly less severe magnitudes in MANU, CONS and PUBL. The selection of active firms operates predominantly in the direction of its mass with the average productivity in TRAN increasing significantly (due to lower $\rho$ ), and blue-collar sectors lose the quantity and quality of firms. Generically, firms entering in the growing sectors are of higher productivity, as the expanding market size favors more productive employers. In particular, higher turnover reduces the risk of bankruptcy and favors high-productivity incumbent firms through raising the cutoff productivity. Opposite effects take place in losing sectors. Finally, the "green" sectors are relatively more capital-intensive, while the "red" sectors 
Figure 6: Scenario 2: Economic Impact of COVID-19 of 2Q2020

(a) Macroeconomic and Labor Market Aggregates

(i) Macro indicators

\begin{tabular}{rrrrrr} 
& \multicolumn{1}{c}{$\mathbf{Y}$} & $\mathbf{P}$ & $\mathbf{L}$ & $\mathbf{M}$ & $\boldsymbol{\varphi}$ \\
\cline { 2 - 6 } MANU & $-15,80 \%$ & $-0,46 \%$ & $-2,70 \%$ & $-19,4 \%$ & $-2,56 \%$ \\
CONS & $-12,05 \%$ & $-1,34 \%$ & $-1,73 \%$ & $-16,7 \%$ & $-2,73 \%$ \\
SALE & $-22,40 \%$ & $-3,40 \%$ & $-2,46 \%$ & $-26,3 \%$ & $-5,83 \%$ \\
\hline TRAN & $12,77 \%$ & $4,00 \%$ & $-1,25 \%$ & $1,92 \%$ & $5,31 \%$ \\
LSER & $-18,43 \%$ & $-2,88 \%$ & $-2,92 \%$ & $-25,4 \%$ & $-0,96 \%$ \\
\hline FSER & $-4,80 \%$ & $-1,78 \%$ & $-0,13 \%$ & $-8,60 \%$ & $0,35 \%$ \\
PSER & $7,51 \%$ & $-0,40 \%$ & $0,87 \%$ & $-0,24 \%$ & $4,57 \%$ \\
\hline PUBL & $-3,19 \%$ & $1,05 \%$ & $-0,94 \%$ & $-10,2 \%$ & $0,58 \%$ \\
Aggr. & $-4,74 \%$ & - & $-1,03 \%$ & $-12,8 \%$ & - \\
\hline
\end{tabular}

(iii) Average Skill Level

\begin{tabular}{rrrrrr}
\cline { 2 - 5 } & \multicolumn{1}{c}{ MAN } & PRO & \multicolumn{1}{c}{ CLC } & \multicolumn{1}{c}{ SER } & ELE \\
\hline \hline MANU & $-6,78 \%$ & $-18,5 \%$ & $-12,0 \%$ & $-3,06 \%$ & $-5,48 \%$ \\
CONS & $8,41 \%$ & $3,99 \%$ & $6,62 \%$ & $-0,80 \%$ & $1,41 \%$ \\
SALE & $-10,42 \%$ & $-5,19 \%$ & $-5,71 \%$ & $-2,95 \%$ & $1,47 \%$ \\
TRAN & $46,50 \%$ & $-0,66 \%$ & $-0,8 \%$ & $1,88 \%$ & $10,30 \%$ \\
LSER & $-1,38 \%$ & $-1,16 \%$ & $3,53 \%$ & $4,22 \%$ & $1,52 \%$ \\
FSER & $-10,39 \%$ & $-0,7 \%$ & $-0,54 \%$ & $-28,0 \%$ & $3,03 \%$ \\
PSER & $10,45 \%$ & $6,17 \%$ & $4,61 \%$ & $-2,73 \%$ & $1,33 \%$ \\
PUBL & $12,20 \%$ & $-0,6 \%$ & $-0,48 \%$ & $1,12 \%$ & $7,96 \%$ \\
\hline \hline
\end{tabular}

(ii) Total Efficient Composite

\begin{tabular}{llrrrrr}
\cline { 2 - 6 } & MAN & PRO & CLC & SER & ELE & UNE \\
\cline { 2 - 6 } MANU & $0,47 \%$ & $-1,83 \%$ & $-2,47 \%$ & $-6,95 \%$ & $-3,60 \%$ & \\
CONS & $2,34 \%$ & $0,49 \%$ & $-0,66 \%$ & $-5,94 \%$ & $-2,97 \%$ & \\
\cline { 2 - 6 } SALE & $0,12 \%$ & $-0,45 \%$ & $-1,86 \%$ & $-6,96 \%$ & $-2,96 \%$ & \\
TRAN & $5,56 \%$ & $0,09 \%$ & $-1,38 \%$ & $-6,58 \%$ & $-2,15 \%$ & \\
LSER & $1,04 \%$ & $-0,04 \%$ & $-0,96 \%$ & $-6,43 \%$ & $-2,96 \%$ & \\
\cline { 2 - 6 } FSER & $0,11 \%$ & $0,00 \%$ & $-1,36 \%$ & $-9,70 \%$ & $-1,12 \%$ & \\
PSER & $2,24 \%$ & $0,71 \%$ & $-0,86 \%$ & $-6,97 \%$ & $-2,97 \%$ & \\
PUBL & $2,75 \%$ & $0,00 \%$ & $-1,35 \%$ & $-6,65 \%$ & $-2,40 \%$ & \\
Aggr. & $2,17 \%$ & $0,83 \%$ & $-0,68 \%$ & $-7,36 \%$ & $-4,37 \%$ & $32,5 \%$ \\
\hline
\end{tabular}

(iv) Number of Employees

\begin{tabular}{|c|c|c|c|c|c|}
\hline & MAN & PRO & CLC & SER & ELE \\
\hline & $10,8 \%$ & $22,03 \%$ & $\bar{~} 13,51 \%$ & $-2,03 \%$ & $4 \overline{4,45}$ \\
\hline DNS & $-2,96 \%$ & $-2,11 \%$ & $-4,63 \%$ & $-3,22 \%$ & $-2,01 \%$ \\
\hline SALE & $14,90 \%$ & $6,36 \%$ & $6,53 \%$ & $-2,1$ & $-2,00$ \\
\hline TRAN & $-25,9 \%$ & $2,06 \%$ & $1,7 \%$ & $-6,41 \%$ & $-9,15 \%$ \\
\hline LSER & $5,32 \%$ & $2,45 \%$ & $-2,08 \%$ & $-8,36 \%$ & $-2,11 \%$ \\
\hline FSER & $14,84 \%$ & $2,0 \%$ & $1,51 \%$ & $28,05 \%$ & $-1,72 \%$ \\
\hline PSER & $-4,84 \%$ & $-3,92 \%$ & $-2,99 \%$ & $-2,39 \%$ & $-1,94 \%$ \\
\hline UBI & $-5,86 \%$ & $1,95 \%$ & $1,46 \%$ & $-5,78 \%$ & $-7,41 \%$ \\
\hline
\end{tabular}

(b) Changes in Aggregated Wage Distributions
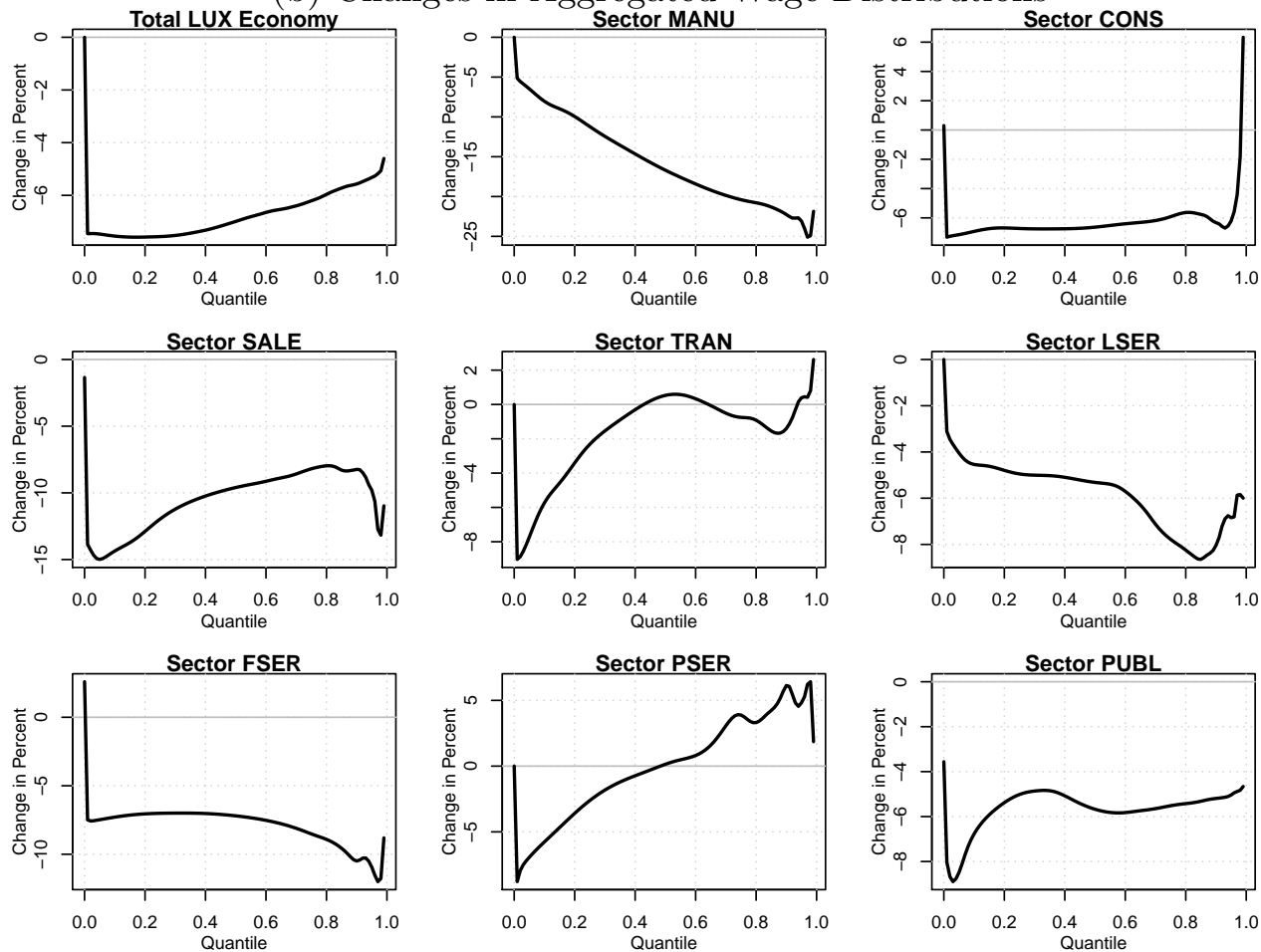

Note: Figures 6a reports changes in (i) key macroeconomic indicators (GDP, price indexes, real production, total labor composite, and the mass of firms), (ii) total efficient labor composites, (iii) average skill levels, and (iv) numbers of employees by $(s, n)$ labor market cells. Figure 6b illustrates relative changes in wage rates for quantiles of respective wage distributions by sectors. 
require higher labor inputs, and are characterized by low values of $\theta_{s}$. Overall, the price, firm selection, and capital-intensity effects contribute to flattening the impact on the labor market: the demand for the efficient labor composite falls by only $1-3 \%$.

Many of the leaving workers upgrade into sectors that grow, as illustrated in panels (ii)-(iv). The most important sectoral moves include upgrading of managers into CONS, TRAN, PSER and PUBL at the expense of MANU, SALE and FSER. Most skilled professionals and clerical workers tend to leave MANU and settle in PSER and CONS, while the ELE occupations switch from MANU to TRAN. As previously discussed, the positions left by people who flow out are taken by workers of lower efficiency, so that the average skill drops in MANU and FSER, while it visibly improves in CONS, TRAN and PSER. We also observe important occupational shifts: the supply of white-collar workers (MAN and PRO) increases in almost all sectors. Both groups are feed with ex-CLC workers, while the majority of people who leave SER and ELE occupations move to unemployment (the equilibrium rate increases by $33 \%$ from 5.6 to 7.4 percent). Accounting for goods' market, firms' and labor market adjustments in a medium-term GE provides an overall drop in nominal GDP at the level of $4.8 \%$. Note that this loss of economy's capacity in generating value added is of permanent nature and cannot be compensated by immanent medium-term economic mechanisms such as capital and labor adjustments or price/wage effects. The only possibility for regaining the loss is to intervene in selected sectors by increasing the demand, reducing employment costs, providing liquidity packages for bankrupting firms or boosting firm creation. Otherwise, the COVID-19 economic slump will cause the Luxembourgish economy to encounter enormous losses.

COVID-19 brings significant wage losses to all employees in Luxembourg, Figure 6b, but once again one observes disproportionately higher burden put on the left tail of the distribution (approximately -8\%), while the top earners lose only around $-5 \%$. Looking at the sectoral decomposition, we can highlight a few patterns that contribute to the overall distributive impact on wages. First, note that highest relative losses take place in those sectors that experience strong negative selection effects: MANU, SALE and FSER. In case of MANU we observe a deterioration in the average skills of the high-earning workers, leading to a $-25 \%$ wage drops for the highest quantiles. In case of SALE and FSER, the mobility of most skilled managers generates losses for the right tail, compensated by gains in CONS, TRAN, and PSER, to where these managers transfer. Gains in the middle quantiles of TRAN wage distribution are mainly caused by the improved selection of the service and elementary occupations. The least skilled workers staying in SALE are another group that experiences extraordinary losses. Remember that their relevant outside options on the labor market are limited for those who are active in blue-collar-intensive sectors, 
chiefly LSER. But LSER is also severely hit by the crisis, and serves as a non-attractive alternative. Therefore, we observe little selection effects - less skilled SALE workers are stuck in their current occupation and are forced to accept huge wage losses (otherwise they join unemployment). Professional services are mostly represented among winners from the COVID-19 crisis. 50\% of the highest earners in PSER observe their wages to grow, up to $5 \%$ relative to status quo. The improved selection of MAN, PRO, and CLC occupations within PSER sector contributes the most, at the expense of FSER, SALE and MANU sectors. This prediction reinforces the inequality-increasing character of the COVID-19 crisis. To sum up, according to our results, the COVID-19 crisis hurts bluecollar workers, while some of the white-collar workers can execute their outside options and escape significant wage losses. These implications for inequality should be seriously reconsidered by policymakers in proposing stimulus packages, as sectoral heterogeneity proves to be significant. Apparently, a uniform provision of grants and economic support across sectors might deepen the distance between workers on the opposite sides of wage distribution.

\section{Concluding Remarks}

In this paper we developed a medium-term approach towards assessing the economic impact of post COVID-19 crisis. We focused on equilibrium changes in prices, wages, allocation of workers across sectors and occupations, and firm dynamics. Further work in this topic could include integrating the fiscal module which would allow for direct policy experiments with firm support, liquidity provisions, and tax cuts. Our model also disregards the long-term impact of the crisis on production technology. Changes in skill prices induced by reallocation of workers might serve as important incentives for firms to substitute certain workers with robots, and reshape the task content of occupations.

\section{References}

Andersen, A. L., Hansen, E. T., Johannesen, N., and Sheridan, A. (2020). Consumer responses to the COVID-19 crisis: Evidence from bank account transaction data. Available at SSRN 3609814.

Arellano-Valle, R. B. and Azzalini, A. (2006). On the unification of families of skew-normal distributions. Scandinavian Journal of Statistics, 33(3):561-574.

Azzalini, A. (1985). A class of distributions which includes the normal ones. Scandinavian Journal of Statistics, pages 171-178. 
Azzalini, A. (2005). The Skew-normal Distribution and Related Multivariate Families. Scandinavian Journal of Statistics, 32(2):159-188.

Azzalini, A. and Valle, A. D. (1996). The Multivariate Skew-Normal Distribution. Biometrika, 83(4):715-726.

Baker, S. R., Farrokhnia, R. A., Meyer, S., Pagel, M., and Yannelis, C. (2020). How does household spending respond to an epidemic? consumption during the 2020 COVID-19 pandemic. Technical report, National Bureau of Economic Research.

Burstein, A., Hanson, G., Tian, L., and Vogel, J. (2020). Tradability and the LaborMarket Impact of Immigration: Theory and Evidence from the United States. Econometrica, 88(3):1071-1112.

Carletti, E., Oliviero, T., Pagano, M., Pelizzon, L., and Subrahmanyam, M. G. (2020). The COVID-19 shock and equity shortfall: Firm-level evidence from Italy.

De Vito, A. and Gomez, J.-P. (2020). Estimating the COVID-19 cash crunch: Global evidence and policy. Journal of Accounting and Public Policy, page 106741.

Demmou, L., Franco, G., Calligaris, S., and Dlugosch, D. (2020). Corporate sector vulnerabilities during the COVID-19 outbreak: assessment and policy responses. VoxEU. org, May, 23.

Gola, P. (2015). Occupational sorting and the structure of status. University of Oxford.

Gola, P. (2020). On the importance of social status for occupational sorting.

Guerini, M., Nesta, L., Ragot, X., and Schiavo, S. (2020). Firm liquidity and solvency under the covid-19 lockdown in france. OFCE Policy Brief, 76.

Heckman, J. J. (1979). Sample Selection Bias as a Specification Error. Econometrica, 47(1):153.

Heckman, J. J. and Honore, B. E. (1990). The Empirical Content of the Roy Model. Econometrica, 58(5):1121.

Heckman, J. J. and Sedlacek, G. (1985). Heterogeneity, Aggregation, and Market Wage Functions: An Empirical Model of Self-Selection in the Labor Market. Journal of Political Economy, 93(6):1077-1125.

Melitz, M. (2003). The Impact of Trade on Aggregate Industry Productivity and IntraIndustry Reallocations. Econometrica, 71(6):1695-1725.

Roy, A. D. (1951). Some Thoughts on the Distribution of Earnings. Oxford Economic Papers, 3(2):135-146.

Simonovska, I. and Waugh, M. E. (2014). The Elasticity of Trade: Estimates and Evidence. Journal of International Economics, 92(1):34-50. 



